ESAIM: M2AN

Vol. 40, No 1, 2006, pp. 201-224

DOI: $10.1051 / \mathrm{m} 2 \mathrm{an}: 2006009$
ESAIM: Mathematical Modelling and Numerical Analysis

www.edpsciences.org/m2an

\title{
A VISCOELASTIC MODEL WITH NON-LOCAL DAMPING APPLICATION TO THE HUMAN LUNGS
}

\author{
Céline Grandmont ${ }^{1},{\text { Bertrand } \text { Maury }^{2} \text { and Nicolas Meunier }}^{3}$
}

\begin{abstract}
In this paper we elaborate a model to describe some aspects of the human lung considered as a continuous, deformable, medium. To that purpose, we study the asymptotic behavior of a spring-mass system with dissipation. The key feature of our approach is the nature of this dissipation phenomena, which is related here to the flow of a viscous fluid through a dyadic tree of pipes (the branches), each exit of which being connected to an air pocket (alvelola) delimited by two successive masses. The first part focuses on the relation between fluxes and pressures at the outlets of a dyadic tree, assuming the flow within the tree obeys Poiseuille-like laws. In a second part, which contains the main convergence result, we intertwine the outlets of the tree with a spring-mass array. Letting again the number of generations (and therefore the number of masses) go to infinity, we show that the solutions to the finite dimensional problems converge in a weak sense to the solution of a wave-like partial differential equation with a non-local dissipative term.
\end{abstract}

Mathematics Subject Classification. 74D05, 74Q10, 76S05, 92B05.

Received: July 1, 2005. Revised: October 30, 2005.

\section{INTRODUCTION, MOTIVATION}

The modelling of air flow in the bronchial tree is made difficult by the complexity of the bronchial net, which rules out the possibility to perform direct simulations on the whole domain. In connection, attempts to provide realistic models for the lungs face the following problem: dissipation effects, which are mainly due to the flow of air through narrow pipes (the branches) is tightly related to the way the air circulates through the tree. The present work is an attempt to address those two issues. From a modelling standpoint, the core of the approach we propose is simply the Poiseuille's law, which relates flow rate and pressure differential for a viscous fluid flowing through a pipe. Note that, as far as the human ventilation system is concerned, this law does not apply to the first generations of branches, where inertial effects cannot be neglected (see [2,9]). We shall nevertheless assume here that this linear law is valid on the overall tree of pipes we consider. Note that physical relevance can be recovered if we consider that the tree we shall introduce in the next section is in fact a subtree (starting at generation 5 or 6 ) of the actual human bronchial system.

Our first step will consist in considering a dyadic net of pipes, through which some viscous fluid flows, and establishing a linear relation between flow rates and pressures at the outlets of the net. We then imbed the

\footnotetext{
Keywords and phrases. Poiseuille flow, dyadic tree, kernel operator, damped wave equation, human lungs.

1 Université Paris Dauphine, 75775 Paris Cedex 16 \& INRIA, France.

${ }^{2}$ Laboratoire de Mathématiques, Université Paris-Sud, 91405 Orsay Cedex, France. bertrand.maury@math.u-psud.fr

${ }^{3}$ Laboratoire Jacques-Louis Lions, Université Pierre et Marie Curie, 75252 Paris Cedex 05, France.
}

(C) EDP Sciences, SMAI 2006 
outlets in an interval of the real line, which makes it possible to have the number of generations go to infinity in a proper mathematical way. The first section focuses on this convergence and on the properties of the limit operator we build.

In the second section, we connect the outlets of the finite trees to a spring-mass array, which is intended to represent the heavy part of the lungs. We let the number of generations go to infinity again, and we establish the convergence of the sequence of solutions to the discrete problems toward a space-time continuous function, which is proved to obey a wave-like equation

$$
\partial_{t t} u-\partial_{x x} u+A\left(\partial_{t} u\right)=f,
$$

where $A\left(\partial_{t} u\right)$ is a non-local damping term ( $A$ is a dissipative operator), which arises from the fluid flow through the tree.

The investigation of the elastic or viscoelastic properties of the lungs has given rise to a great number of papers during the last two decades. Some authors base their approach on discrete spring-mass systems (see $[3,4,8,12])$. Others have proposed constitutive equations at the continuous level (see $[5,13])$. To our knowledge, the present work is the first attempt to incorporate, by mean of a rigorous homogeneization procedure, non-local effects due to fluid circulation in the constitutive equations of an organic medium.

\section{Flow through a dyadic tree}

We consider here a viscous fluid which flows through a tree of connected pipes, each of which being characterized by its resistance (ratio between the pressure jump between its ends and the flux). Our first step will consist in establishing the relation between pressures and fluxes at the outlets, assuming a dyadic structure of the tree. We shall then investigate the possible convergence (in a sense which will be detailed further) of those relations toward some generalized porous medium models, when the height of the tree goes to infinity.

\subsection{Model problem}

We consider the flow of an incompressible, viscous, non-inertial, fluid through a pipe. The pressure is supposed to be uniform over each end section of the pipe (to which we shall refer as 0 and 1 ), so that the external forces acting on the fluid can be characterized by two values $P_{0}$ and $P_{1}$. The linearity of the Stokes equations ensures the existence of a coefficient $r>0$ which relates the flux $Q$ (considered positive if the fluid goes from 0 to 1 ) and the pressure jump $P_{1}-P_{0}$

$$
P_{1}-P_{0}=-r Q .
$$

By analogy with electric conductors (flux and pressure play the roles of intensity and potential, respectively), $r$ is called the resistance of the pipe. It depends on geometrical characteristics of the pipe and on the viscosity of the fluid.

We consider now a full dyadic tree of such pipes. The height of the tree is denoted by $N+1$. We suppose that pipes which belong to the same generation $n$ have a common resistance $r_{n}>0$. Note that most of the abstract properties we establish can be extended to the case of non-constant resistances (see Rem. 1.12). This feature is important as we intend to apply in the future this approach to the modelling of some pulmonary affections like asthma, which is known to perturb the homogeneity of inner diameters of branches. Nevertheless, as explicit expressions are much more complicated to get with non-constant resistances, we shall restrict ourselves to this simplified framework. We shall suppose that the pressure at the root node is set at pressure 0 , and we shall denote by $q_{i}$ and $p_{i}$, respectively, the flux and pressure corresponding to the outlet $i$, for $i=0, \ldots, 2^{N}-1$. Given a set of pressures (resp. a set of fluxes), it is possible to compute the corresponding set of fluxes (resp. pressures). As $N$ goes to infinity, supposing the outlets (i.e. leafs of the tree) tend to cover a given domain $\Omega$ of the physical space $\mathbb{R}^{d}$, we propose to investigate whether the aforementioned correspondence between pressure and fluxes can tend to something at the continuous level. We shall restrict ourselves in the present approach to the case $d=1$. 


\subsection{Solution for the finite tree}

Outlets of the tree will be indexed by $0,1, \ldots, 2^{N}-1$. Let

$$
\mathbf{p}=\left(p_{i}\right)_{i=0, \ldots, 2^{N}-1} \text { and } \mathbf{q}=\left(q_{i}\right)_{i=0, \ldots, 2^{N}-1}
$$

be the pressure and flux vectors at the outlets of the tree. Fluxes are considered positive when the fluid enters the tree.

Definition 1.1. Given two positive integers $i$ and $j$ and their binary expressions

$$
i=\sum_{k=0}^{\infty} \alpha_{k} 2^{k}, j=\sum_{k=0}^{\infty} \beta_{k} 2^{k} \text { with } \alpha_{k}, \beta_{k} \in\{0,1\} \quad \forall k
$$

we define $\nu_{i j}$ as

$$
\nu_{i j}=\inf \left\{k \geq 0, \alpha_{\ell}=\beta_{\ell} \quad \forall \ell \geq k\right\} .
$$

Proposition 1.2. We consider a full dyadic tree characterized by its generation-wise resistances $r_{0}, r_{1}, r_{2}, \ldots$, $r_{N}$. Supposing that the root node is at pressure 0 , then pressures and fluxes at the outlets are related by

$$
\mathbf{p}=A^{N} \mathbf{q}, A^{N}=\left(A_{i j}^{N}\right)_{0 \leq i, j \leq 2^{N}-1} \in \mathcal{M}_{2^{N}}(\mathbb{R}), A_{i j}^{N}=R_{N-\nu_{i j}}
$$

where $R_{n}$ is the cumulated resistance $r_{0}+r_{1}+\cdots+r_{n}$.

Proof. The linearity of the relation between $\mathbf{q}$ and $\mathbf{p}$ is obvious. Hence it is sufficient to compute the pressure vectors associated to the elements of the canonical basis of $\mathbb{R}^{2^{N}}$. As a first step, we consider $\mathbf{q}=(1,0,0, \ldots, 0)$. It corresponds to the situation where some fluid flows through outlet 0 (node $X_{N}$ in Fig. 1), and only this one. By conservation, the flux which exits the domain through the root is exactly 1 , so that the pressure at node $X_{0}$ (see Fig. 1) is $r_{0}$. As there is no flow in the right-hand subtree stemming from $X_{0}$, the pressure at its leafs (outlets with indices between $2^{N-1}$ and $2^{N}-1$ ) is exactly $r_{0}$. Similarly, the pressure at node $X_{1}$ is $r_{0}+r_{1}=R_{1}$, and so is the pressure at the outlet of the right-hand subtree stemming from $X_{1}$. Following this approach recursively, one finds pressures at nodes $X_{2}, X_{3}, \ldots, X_{N}$ to be $R_{2}, R_{3}, \ldots, R_{N}$, respectively. Consequently, the pressure at outlet $j$ is $R_{N-\nu_{0 j}}$. The same reasoning can be applied to any vector $\mathbf{q}=(0, \ldots, 0,1,0, \ldots, 0)$ (with the 1 at position $i$ ), to which corresponds the pressure field

$$
\left(R_{N-\nu_{i 0}}, R_{N-\nu_{i 1}}, \ldots, R_{N-\nu_{i 2}-1}\right) .
$$

We thus obtain

$$
p_{i}=\sum_{j=0}^{2^{N}-1} q_{j} R_{N-\nu_{i j}}
$$

which ends the proof.

Remark 1.3. The resistance matrix $A^{N} \in \mathcal{M}_{2^{N}}(\mathbb{R})$ is, up to a multiplicative constant, a doubly stochastic matrix: all raws and columns have a common sum, which will be shown to be the global resistance of the tree considered as a single conductor.

\subsection{Limit as $N$ goes to $+\infty$}

We shall limit ourself in the present approach to the case where $\Omega$ is the interval $I=] 0,1[$. Our first step will consist in giving a sense to the discrete problem at the continous level. The approach is the following. Given a height $N>0$, we identify the outlet node $i$ of the tree $T_{N}$ to the segment $] i h_{N},(i+1) h_{N}\left[\subset I\right.$, with $h_{N}=1 / 2^{N}$. It makes it possible to define the following operator. 


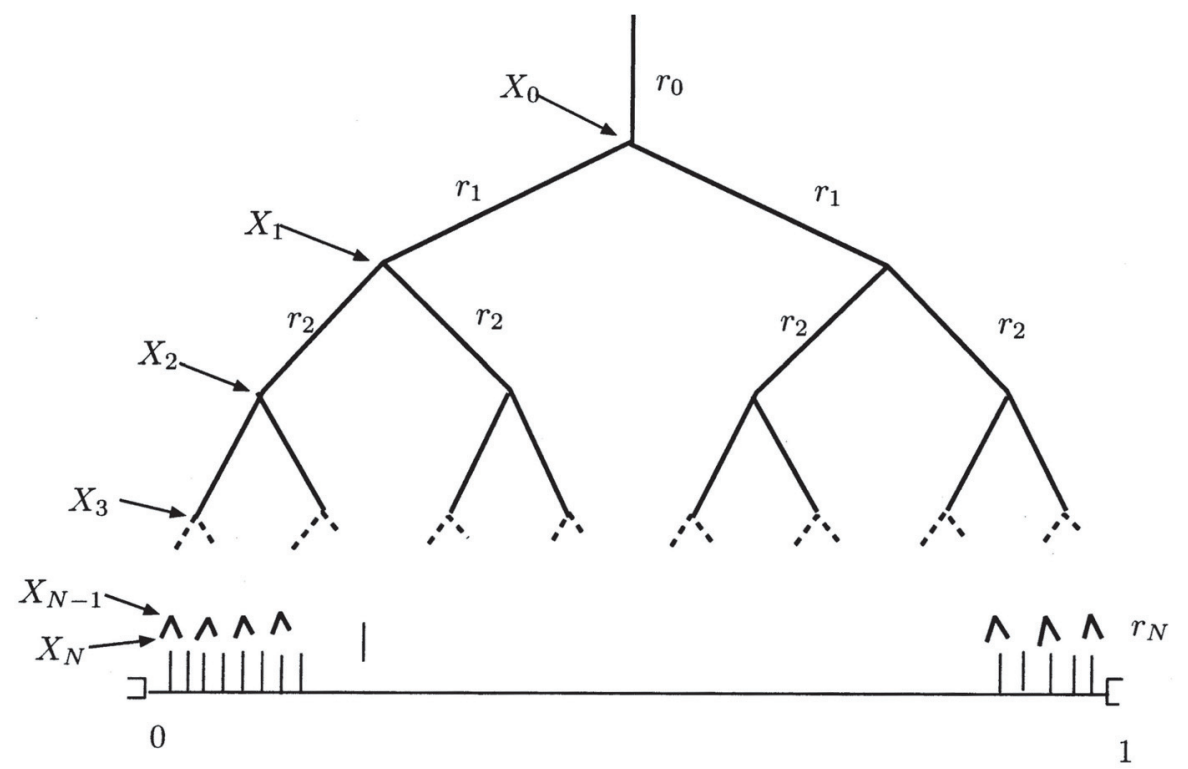

Figure 1. Dyadic tree.

Definition 1.4. Given a function $u \in L^{2}(I)$, we define the flux vector $\mathbf{q}^{N}$ as

$$
\mathbf{q}^{N}=\left(q_{i}^{N}\right)_{i=0, \ldots, 2^{N}-1}, q_{i}^{N}=\int_{i h_{N}}^{(i+1) h_{N}} u(y) \mathrm{d} y .
$$

The corresponding discrete pressure vector is defined as

$$
\mathbf{p}^{N}=\left(p_{i}^{N}\right)_{i=0, \ldots, 2^{N}-1}=A^{N} \mathbf{q}^{N}
$$

where $A^{N} \in \mathcal{M}_{2^{N}}(\mathbb{R})$ is the matrix which expresses relation (4). Finally, we define the function $p \in L^{2}(I)$ as the function which takes constant value $p_{i}^{N}$ in each subinterval $] i h_{N},(i+1) h_{N}[$. The corresponding operator, which maps $L^{2}(I)$ onto itself, will be denoted by $\mathcal{R}_{N}$.

Proposition 1.5. The operator $\mathcal{R}_{N}$ can be expressed as an integral operator:

$$
p=\mathcal{R}_{N} u \Longleftrightarrow p(x)=\int_{I} K_{N}(x, y) u(y) \mathrm{d} y \quad \text { a.e. }
$$

where $K_{N} \in L^{1}(I \times I)$ is a piecewise constant function defined as

$$
x \in] i h_{N},(i+1) h_{N}[, y \in] j h_{N},(j+1) h_{N}\left[\longmapsto R_{N-\nu_{i j}} .\right.
$$


Proof. By definition, the pressure vector $\mathbf{p}^{N}$ is $A^{N} \mathbf{q}^{N}$, where $A^{N}$ is the matrix associated with the linear mapping expressed by (4), so that

$$
\begin{aligned}
p_{i}^{N} & =\sum_{j=0}^{2^{N}-1} q_{j}^{N} R_{N-\nu_{i j}} \\
& =\sum_{j=0}^{2^{N}-1} R_{N-\nu_{i j}} \int_{j h_{N}}^{(j+1) h_{N}} u(y) \mathrm{d} y \\
& =\sum_{n=0}^{N-1} R_{N-n} \sum_{0 \leq j \leq 2^{N}-1, \nu_{i j}=n} \int_{j h_{N}}^{(j+1) h_{N}} u(y) \mathrm{d} y .
\end{aligned}
$$

Finally, for (almost) every $x \in I, x \in] i h_{N},(i+1) h_{N}[$,

$$
p^{N}(x)=p_{i}^{N}=\int_{I} K_{N}(x, y) u(y) \mathrm{d} y,
$$

by definition of $K_{N}$.

Proposition 1.6. The kernel $K_{N}$ converges almost everywhere to the measurable function $K$ defined as follows: Let $x$ and $y$ be given in $I$, with dyadic expressions

$$
x=\sum_{k=0}^{\infty} \frac{\alpha_{k}}{2^{k}}, y=\sum_{k=0}^{\infty} \frac{\beta_{k}}{2^{k}} .
$$

We define (note that $\alpha_{0}=\beta_{0}=0$ )

$$
\mu_{x y}=\sup \left\{k \geq 1, \alpha_{\ell}=\beta_{\ell} \quad \forall \ell<k\right\}
$$

If one of the sequences $\left(\alpha_{k}\right),\left(\beta_{k}\right)$ has a finite number of non-zero terms, or if $x=y$, we set $K(x, y)=0$. Otherwise, $K(x, y)$ is set to $R_{\mu_{x y}}$.

Proof. Let us remark that $K_{N}$ and $K_{N-1}$ are related by

$$
K_{N}(x, y)=K_{N-1}(x, y)+r_{N} \sum_{i=0}^{2^{N}-1} \chi_{i}^{N}(x, y)
$$

where $\chi_{i}^{N}$ is defined as the characteristic function of the square

$$
] i h_{N},(i+1) h_{N}[\times] i h_{N},(i+1) h_{N}[\subset I \times I .
$$

As a consequence, for any extra-diagonal couple $(x, y)(i . e$. with $x \neq y),\left(K_{N}(x, y)\right)_{N}$ is stationary as soon as $N>-\log _{2}|y-x|$.

Theorem 1.7. The sequence $\left(\mathcal{R}_{N}\right)$ converges to a limit $\mathcal{R}$ in $\mathcal{L}\left(L^{2}(I)\right)$ if and only if

$$
\sum_{n=0}^{+\infty} \frac{r_{n}}{2^{n}}<+\infty
$$




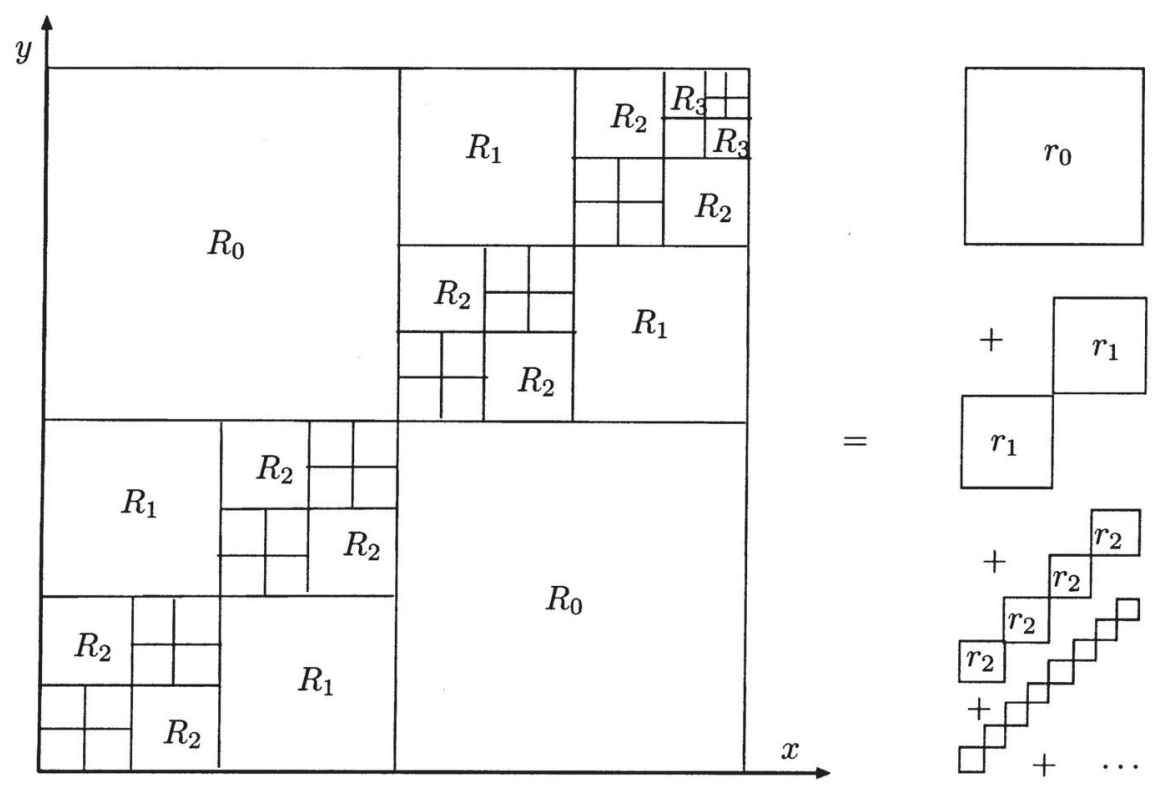

Figure 2. Kernel $K(x, y)$.

When it exists, the limit $\mathcal{R}$ is an integral operator with associated kernel

$$
K=\lim _{N \rightarrow+\infty} K_{N} \text { in } L^{1}(I \times I) .
$$

Proof. Firstly, consider the function $u$ which is identically equal to 1 over $I$. Then $\mathcal{R}_{N}(u)$ is itself constant over $I$, and its value is (here $x$ designs any real number in $I$ which is not of the form $k / 2^{N}$ )

$$
p=\int_{I} K_{N}(x, y) \mathrm{d} y=\sum_{n=0}^{N} \frac{r_{n}}{2^{n}}
$$

which shows that (7) is a necessary condition. Assume now that condition (7) holds. The function $K$ defined in Proposition 1.6 is then in $L^{1}(I \times I)$. Indeed,

$$
\iint|K(x, y)| \mathrm{d} x \mathrm{~d} y=\sum_{N=0}^{+\infty} r_{N} \sum_{i=0}^{2^{N}-1} \iint \chi_{i}^{N}(x, y) \mathrm{d} x \mathrm{~d} y=\sum_{n=0}^{+\infty} \frac{r_{n}}{2^{n}}<+\infty
$$

By the dominated convergence theorem, $\left(K_{N}\right)_{N}$ converges therefore to $K$ in $L^{1}(I \times I)$. Noting that $\left\|K_{N}\right\|_{L_{x}^{1}(I)}=$ $\left\|K_{N}\right\|_{L^{1}(I \times I)}$ it is easy to verify that for all $u \in L^{p}(I), 1 \leq p \leq \infty$,

$$
\left\|\mathcal{R}_{N} u\right\|_{L^{p}(I)} \leq\left\|K_{N}\right\|_{L^{1}(I \times I)}\|u\|_{L^{p}(I)} .
$$

This "convolution" type inequality can be proved following the same steps as in [1], Th IV. 15. As a consequence, the mapping $K_{N} \mapsto \mathcal{R}_{N}$ is continuous from $L^{1}(I \times I)$ onto $\mathcal{L}\left(L^{2}(I)\right)$, and it implies the convergence in the operator norm of $\mathcal{R}_{N}$ to $\mathcal{R}$ defined as

$$
\mathcal{R} u(x)=\int_{I} K(x, y) u(y) \mathrm{d} y
$$


Proposition 1.8. If condition (7) holds, i.e. $\mathcal{R}_{N}$ converges, the limit operator $\mathcal{R}$ is self-adjoint, compact, and monotone.

Proof. As $K(x, y)=K(y, x)$, the integral operator $\mathcal{R}$ is self-adjoint. Furthermore, as $\mathcal{R}_{N} u$ is piecewise constant with respect to the subdivision $0, h_{N}, 2 h_{N}, \ldots, 1$, the operator $\mathcal{R}_{N}$ has finite rank. Therefore $\mathcal{R}$ is compact as a limit of a sequence of finite rank operators.

Finally, from relation $(6)$,

$$
(\mathcal{R} u, u)=\sum_{N=0}^{+\infty} r_{N} \sum_{j=0}^{2^{N}-1}\left(\int_{j h_{N}}^{(j+1) h_{N}} u(x) \mathrm{d} x\right)^{2} \geq 0
$$

In the case where $\sum r_{n} / 2^{n}=+\infty$, the kernel $K_{N}$ concentrates in the neighborhood of the diagonal of $I \times I$, and we have the following result:

Proposition 1.9. For all $q \in L^{2}(I)$, the following convergence holds true

$$
\int_{I} \tilde{K}_{N}(x, \cdot) q(x) \mathrm{d} x \longrightarrow q(\cdot) \text { strongly in } L^{2}(I),
$$

where $\tilde{K}_{N}=K_{N} /\left\|K_{N}\right\|_{L^{1}(I \times I)}$.

Proof. We recall that $\left\|K_{N}\right\|_{L^{1}(I \times I)}=\left\|K_{N}\right\|_{L_{x}^{1}(I)}=\sum_{n=0}^{N} \frac{r_{n}}{2^{n}}$. Consequently

$$
\int_{I} \tilde{K}_{N}(x, y) q(x) \mathrm{d} x-q(y)=\int_{I} \tilde{K}_{N}(x, y)(q(x)-q(y)) \mathrm{d} x .
$$

Due to the definition of the kernel $K_{N}$ and the fact that $\left(K_{N}(x, y)\right)_{N}$ is stationary as soon as $N>-\log _{2}|x-y|$, we have that for all $\eta>0$, for all $\varepsilon>0$, there exists $N_{0} \in \mathbb{N}$ such that $\forall(x, y) \in I \times I,|x-y|>\varepsilon, \forall N \geq N_{0}$ $\left|\tilde{K}_{N}(x, y)\right|<\eta$. Thus, considering in a first step $q \in C^{0}(\bar{I})$ we obtain that for all $\eta>0$ there exist $\varepsilon>0$ and $N_{0} \in \mathbb{N}$ such that $\forall N \geq N_{0}$ :

$$
\begin{aligned}
\left|\int_{I} \tilde{K}_{N}(x, y) q(x) \mathrm{d} x-q(y)\right| & \leq\left|\int_{|x-y|>\varepsilon} \tilde{K}_{N}(x, y)(q(x)-q(y)) \mathrm{d} x\right|+\left|\int_{|x-y|<\varepsilon} \tilde{K}_{N}(x, y)(q(x)-q(y)) \mathrm{d} x\right| \\
& \leq C \eta+\left\|\tilde{K}_{N}\right\|_{L_{x}^{1}(I)} \sup _{x,|x-y|<\varepsilon}|q(x)-q(y)| \leq C \eta
\end{aligned}
$$

The lemma holds true for $q \in C^{0}(\bar{I})$ and remains true for $q \in L^{2}(I)$ by a density argument. Indeed let us consider $q \in L^{2}(I)$ and $q_{1} \in C^{0}(\bar{I})$ such that $\left\|q-q_{1}\right\|_{L^{2}(I)} \leq \eta$. The desired result comes from the fact that

$$
\left\|\int_{0}^{1} \tilde{K}_{N}(x, y)\left(q(x)-q_{1}(x)\right) \mathrm{d} x\right\|_{L_{y}^{2}(I)} \leq\left\|\tilde{K}_{N}\right\|_{L_{x}^{1}(I)}\left\|q-q_{1}\right\|_{L^{2}(I)},
$$

and $\left\|\tilde{K}_{N}\right\|_{L_{x}^{1}(I)}=1$.

The spectral decomposition of the limit operator $\mathcal{R}$ turns out to be straightforward, as the so called Haar basis of $L^{2}(I)$ is a basis of eigenvectors for $\mathcal{R}$.

Definition 1.10 (Haar basis). Let $\Psi \in L^{2}(I)$ be defined as $\Psi=1_{] 0,1 / 2[}-1_{] 1 / 2,1[}$. For any $N \geq 0,0 \leq k \leq 2^{N}-1$, we introduce

$$
x \in I \longmapsto \Psi_{N, k}(x)=2^{N / 2} \Psi\left(2^{N} x-k\right),
$$

and we define $\Psi_{0}$ by $\Psi_{0}(x) \equiv 1$. The family $\left(\Psi_{0},\left(\Psi_{N, k}\right)_{0 \leq N, 0 \leq k \leq 2^{N}-1}\right)$ is a Hilbert basis of $L^{2}(I)$, called the Haar basis. 
Proposition 1.11. The Haar basis $\left(\Psi_{0},\left(\Psi_{N, k}\right)_{0 \leq N}, 0 \leq k \leq 2^{N}-1\right)$ is a family of eigenvectors for $\mathcal{R}$. The corresponding family of eigenvalues is $\left(\lambda_{0},\left(\lambda_{N, k}\right)_{0 \leq N}, 0 \leq k \leq 2^{N}-1\right)$, with

$$
\lambda_{N, k}=\rho_{N+1}=\sum_{\ell=N+1}^{+\infty} \frac{r_{\ell}}{2^{\ell}} \quad 0 \leq N, 0 \leq k \leq 2^{N}-1
$$

and $\lambda_{0}=\rho_{0}=\sum_{\ell=0}^{+\infty} \frac{r_{\ell}}{2^{\ell}}$ is the global resistance.

Proof. This property can be checked straightforwardly from Figure 2. More formally, for any $N \geq 0,0 \leq k \leq$ $2^{N}-1$, one can express (with $h_{N}=1 / 2^{N}$ )

$$
\begin{aligned}
\mathcal{R} \Psi_{N, k}(x) & =\int_{I} K(x, y) \Psi_{N, k}(y) \mathrm{d} y=\sum_{j=0}^{2^{N}-1} \int_{j h_{N}}^{(j+1) h_{N}} K(x, y) \Psi_{N, k}(y) \mathrm{d} y \\
& =\int_{k h_{N}}^{(k+1) h_{N}} K(x, y) \Psi_{N, k}(y) \mathrm{d} y \quad \text { because } \operatorname{supp}\left(\Psi_{N, k}\right)=\left[k h_{N},(k+1) h_{N}\right] .
\end{aligned}
$$

For any generic $x$ (i.e. not of the form $j h_{N}$ ) which is not in $] k h_{N},(k+1) h_{N}[$, the function $K(x, \cdot)$ is constant over this subinterval, and therefore ( $\Psi_{N, k}$ has zero mean value over this interval) the latter expression is zero. For $x$ in $] k h_{N},(k+1 / 2) h_{N}[$, a straightforward computation gives

$$
\int_{k h_{N}}^{(k+1) h_{N}} K(x, y) \Psi\left(2^{N} y-k\right) \mathrm{d} y=\sum_{\ell=N+1}^{+\infty} \frac{r_{\ell}}{2^{\ell}}
$$

and we find an opposite value for $x$ in $](k+1 / 2) h_{N},(k+1) h_{N}[$, so that

$$
\mathcal{R} \Psi_{N, k}=\lambda_{N, k} \Psi_{N, k} \quad \text { with } \quad \lambda_{N, k}=\rho_{N+1}=\sum_{\ell=N+1}^{+\infty} \frac{r_{\ell}}{2^{\ell}} .
$$

As for the constant eigenfunction $\Psi_{0}$ the eigenvalue is simply the global resistance of the tree $\rho_{0}$.

Remark 1.12. In case we no longer assume that the resistances are uniform within each generation, one can derive convergence results on the corresponding kernel $K_{N}$ and on the resulting operator $\mathcal{R}_{N}$. Note however that the eigenfunctions and eigenvalues of $\mathcal{R}$ are not so easy to compute. Denoting by $\left(r_{n}^{i}\right)_{1 \leq i \leq 2^{n}}$ the resistances at the $n$th generation, under the condition

$$
\sum_{n=0}^{+\infty} \sum_{i=1}^{2^{n}} \frac{r_{n}^{i}}{2^{2 n}}<+\infty
$$

$K_{N}$ converges in $L^{1}(I \times I)$,

and under the sufficient condition

$$
\sum_{n=0}^{+\infty} \frac{\max _{i} r_{n}^{i}}{2^{n}}<+\infty
$$

the associated operator $\mathcal{R}_{N}$ converges in $\mathcal{L}\left(L^{2}(I)\right)$. 


\subsection{Geometric tree}

We consider in this section the case where resistances follow the geometric law

$$
r_{n}=r_{0} \alpha^{n}
$$

where $\alpha$ is a parameter. We assume $\alpha \in] 0,2\left[\right.$, so that $\mathcal{R}$ is properly defined as an operator from $L^{2}(I)$ onto itself. According to the previous section, this operator is symmetric, compact, monotone, and its eigenelements are

$$
\Psi_{0} \text { with eigenvalue } \lambda_{0}=\rho_{0}=\frac{r_{0}}{1-\alpha / 2}, \Psi_{N, k} \text { with eigenvalue } \lambda_{N, k}=\rho_{0}\left(\frac{\alpha}{2}\right)^{N+1}
$$

Firstly, in the case of a geometric behaviour of the resistances, we can express the rate of convergence of the operator $\mathcal{R}_{N}$ towards $\mathcal{R}$ as $N$ goes to infinity.

Proposition 1.13. We suppose $r_{n}=r_{0} \alpha^{n}$, with $\left.\alpha \in\right] 0,2\left[\right.$. Let $\mathcal{R}_{N}$ be defined as in Proposition 1.5, and $\mathcal{R}$ as in Theorem 1.7. We have

$$
\left\|\mathcal{R}-\mathcal{R}_{N}\right\|_{\mathcal{L}\left(L^{2}(I)\right)} \leq \rho_{0}\left(\frac{\alpha}{2}\right)^{N+1}
$$

Proof. Thanks to the convolution type inequality satisfied by $\mathcal{R}-\mathcal{R}_{N}$ we have, for any $u \in L^{2}(I)$,

$$
\left\|\mathcal{R} u-\mathcal{R}_{N} u\right\|_{L^{2}(I)} \leq\left\|K-K_{N}\right\|_{L^{1}(I \times I)}\|u\|_{L^{2}(I)} \leq r_{0} \sum_{n=N+1}^{\infty}\left(\frac{\alpha}{2}\right)^{n}\|u\|_{L^{2}(I)} \leq \rho_{0}\left(\frac{\alpha}{2}\right)^{N+1}\|u\|_{L^{2}(I)} .
$$

Remark 1.14. A similar estimate can be obtained in the general case: The quantity $\left\|\mathcal{R}-\mathcal{R}_{N}\right\|_{\mathcal{L}\left(L^{2}(I)\right)}$ is simply bounded by the eigenvalue $\rho_{N+1}$.

Next, the geometric behavior of the resistances makes it possible to evaluate the singularity of the kernel $K$ in the neighbourhood of the diagonal.

Proposition 1.15. We suppose $r_{n}=r_{0} \alpha^{n}$, with $\alpha>0$, and $K$ is the function defined in Proposition 1.6. If $\alpha \in] 0,1[$, the kernel $K$ is bounded. For $\alpha=1$

$$
0 \leq K(x, y) \leq-C \ln |x-y|
$$

and for $\alpha \in] 1,+\infty[$,

$$
0 \leq K(x, y) \leq C|x-y|^{-\ln \alpha / \ln 2} .
$$

Proof. This is a direct consequence of

$$
|x-y| \in] \frac{1}{2^{N+1}}, \frac{1}{2^{N}}\left[\Longrightarrow K(x, y) \leq R_{N}=r_{0} \sum_{n=0}^{N} \alpha^{n}\right.
$$

which behaves like $N$ for $\alpha=1$, and like $\alpha^{N}$ for $\alpha>1$. The case $\alpha<1$ is straightforward.

As another direct consequence of the expression of $K_{N}$, we have the following properties.

Corollary 1.16. Depending on the value of $\alpha$, the kernel $K$ verifies

$$
\begin{array}{rll}
0 \leq \alpha<1 & \Longrightarrow K \in L^{\infty}(I \times I) & \\
\alpha=1 & \Longrightarrow K \in L^{p}(I \times I) \quad \forall p \in[1,+\infty[ \\
1<\alpha<2 \quad \Longrightarrow K \in L^{q}(I \times I) \quad \forall q<\ln 2 / \ln \alpha .
\end{array}
$$


Proposition 1.17. We suppose $r_{n}=r_{0} \alpha^{n}$, with $\left.\alpha \in\right] \sqrt{2}, 2\left[\right.$. Then $\mathcal{R}$ maps continuously $L^{2}(I)$ onto $H^{s}(I)$, with $s=1-\frac{\ln \alpha}{\ln 2}$.

Proof. This property is a direct consequence of the following characterization of $H^{s}(I)$, for $s \in[0,1 / 2[$ (see e.g. [11]) :

$$
H^{s}(I)=\left\{u \in L^{2}(I),\left|c_{0}(u)\right|^{2}+\sum_{N=0}^{\infty} \sum_{k=0}^{2^{N}-1} 2^{2 s N}\left|c_{N, k}(u)\right|^{2}<\infty\right\}
$$

where $c_{0}(u)$ and the $c_{N, k}(u)$ 's are the coefficients of the expansion of $u$ in the Haar basis,

$$
c_{0}(u)=\int_{I} u(x) \Psi_{0}(x) \mathrm{d} x, c_{N, k}(u)=\int_{I} u(x) \Psi_{N, k}(x) \mathrm{d} x .
$$

We endow $H^{s}$ with the norm

$$
\|p\|_{s}^{2}=\left|c_{0}(u)\right|^{2}+\sum_{N=0}^{\infty} \sum_{k=0}^{2^{N}-1} 2^{2 s N}\left|c_{N, k}(p)\right|^{2}
$$

For any $u \in L^{2}(I)$,

$$
p=\mathcal{R} u=\rho_{0} c_{0}(u) \Psi_{0}+\rho_{0} \sum_{N=0}^{\infty} \sum_{k=0}^{2^{N}-1}\left(\frac{\alpha}{2}\right)^{N+1} c_{N, k}(u) \Psi_{N, k}=c_{0}(p) \Psi_{0}+\sum_{N=0}^{\infty} \sum_{k=0}^{2^{N}-1} c_{N, k}(p) \Psi_{N, k},
$$

with

$$
2^{2 s N}\left|c_{N, k}(p)\right|^{2}=\rho_{0}^{2} 2^{2 s N}\left(\frac{\alpha}{2}\right)^{2 N+2}\left|c_{N, k}(u)\right|^{2} \leq \rho_{0}^{2}\left|c_{N, k}(u)\right|^{2}
$$

as soon as $s=1-\ln (\alpha) / \ln (2)$. For this value of $\alpha$, we therefore have $p \in H^{s}(I)$ by characterization (9).

Remark 1.18. As $\alpha$ drops below $\sqrt{2}$, the range of $\mathcal{R}$ can no longer be identified with the Sobolev space $H^{s}(I)$, for $s=1-\ln \alpha / \ln 2$, because this range contains functions which are discontinuous. Note that, for $\alpha<\sqrt{2}$, the kernel $K$ is in $L^{2}(I \times I)$, so that $\sqrt{2}$ is also the value under which $\mathcal{R}$ becomes a Hilbert-Schmidt operator.

The dual counterpart of the previous proposition, which is again a direct consequence of the characterization (9), can be expressed in the following way:

Proposition 1.19. We suppose $\alpha \in] 1,2\left[\right.$. The complete closure of $\|u\|_{\mathcal{R}}=(\mathcal{R} u, u)^{1 / 2}$ is $H^{s}(I)$, with $s=$ $-1 / 2+\frac{\ln \alpha}{2 \ln 2}<0$.

\subsection{Some remarks on the actual respiration tree}

The human lung can be considered as a full finite binary tree of height 23. According to the literature (see e.g. [10]), the size ratio between a generation and the following is close to $\lambda=0.85$. In reality, the flow of the air through the branches can be considered as linear (i.e. it obeys the Stokes equations) beyond generation 5 or 6 only. The flow in the upper part of the tree follows the Navier-Stokes equations (see [9]). Nevertheless, as our approach focuses on the asymptotic behaviour as the number of generations goes to infinity, we can apply it to the whole respiratory system, simply keeping in mind that the model is not valid for the first generations. From Poiseuille's law, which gives the resistance of a circular pipe as a function of its dimensions, it implies that resistances obey the following law

$$
r_{n}=r_{0} \alpha^{n},
$$

with $\alpha=\lambda^{-3} \sim 1.63$. The actual tree is then convergent in the sense that the sequence $\left(\mathcal{R}_{N}\right)$ converges to $\mathcal{R} \in \mathcal{L}\left(L^{2}\right)$, in the case $\alpha=1.63$. To express the speed of convergence, and to advocate the use of an infinite tree in place of the real tree (which is 23 generations long), let us simply mention that, for $N=23$, the global equivalent resistance is reached with a relative accuracy of $1 \%$. The range of $\mathcal{R}$ is $H^{s}(I)$ for $s \sim 0.3$. 


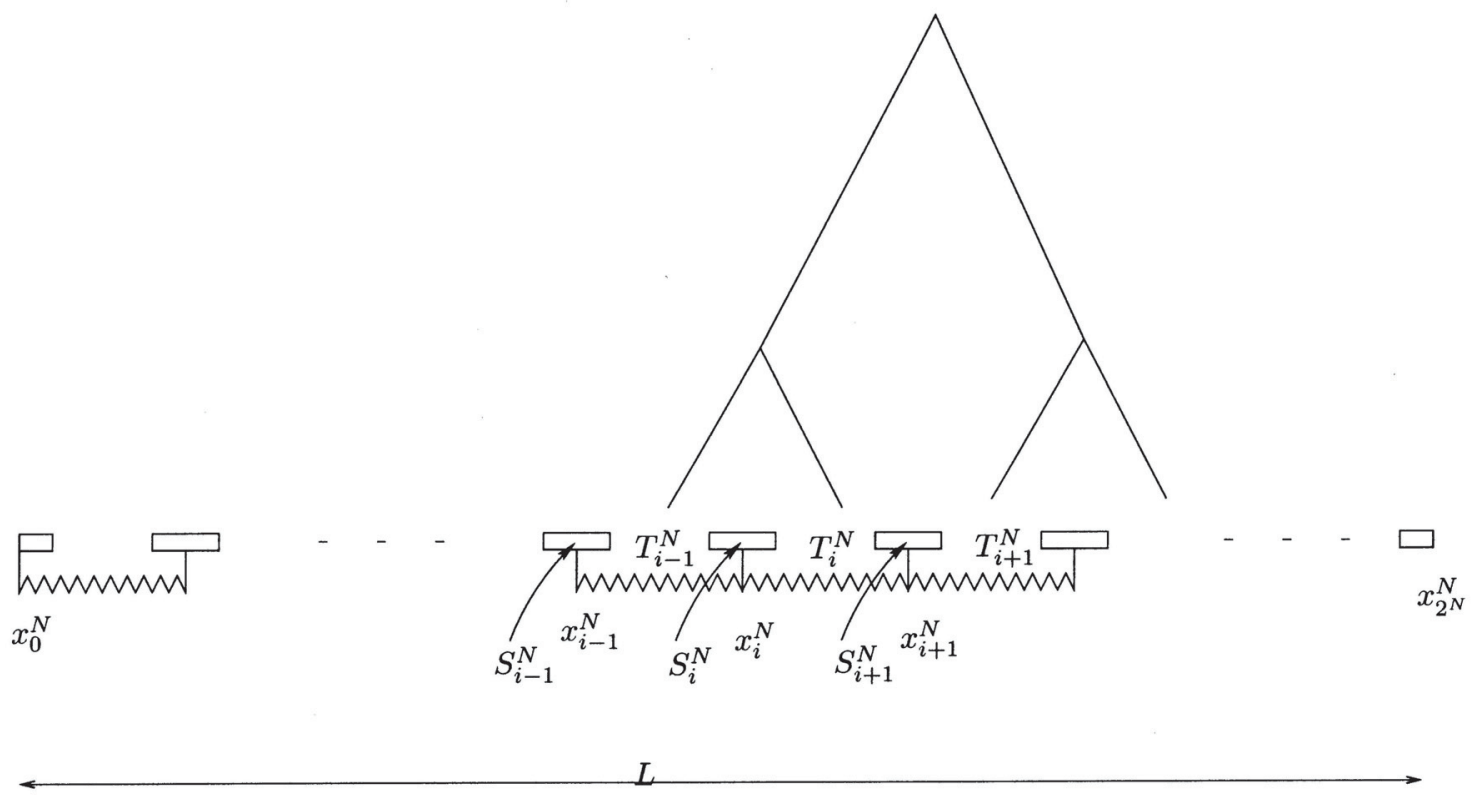

FiguRE 3. Mass-spring-tree system.

\section{SPRING-MASS SYSTEM}

In this section we investigate a one dimensional model of the parenchyma that is a succession of air pockets (alveoli) and masses (lung tissue). The masses are linked by springs and we assume that all springs have the same stiffness. The air can escape through the dyadic tree of pipes we presented in the previous section. Thus each air pocket is connected to each other through the tree. Our purpose is to study the limit behavior of this system when the number of masses goes to infinity.

\subsection{Discrete model}

Let $\Omega=] 0, L$ [ be a succession of masses and air pockets each of which are connected through a dyadic tree of pipes (see Fig. 3). Let $N$ be the number of generations of this finite tree. Since there are $2^{N}$ leafs in the tree we define $2^{N}$ cells

$$
\left.\Omega=\cup_{i=0}^{2^{N}-1} C_{i}^{N}, \text { with } C_{i}^{N}=\right] i h_{N},(i+1) h_{N}\left[\text {, and } h_{N}=\frac{L}{2^{N}} .\right.
$$

Furthermore we set $x_{i}^{N}=i h_{N}$, for all $i \in\left\{0, \ldots, 2^{N}\right\}$, and we will denote by $S_{i}^{N}$ (resp. $T_{i}^{N}$ ) the solid (resp. air) parts:

$$
\begin{gathered}
\left.S_{i}^{N}=\right] x_{i}^{N}-\frac{1}{2} h_{N} \alpha_{S}, x_{i}^{N}+\frac{1}{2} h_{N} \alpha_{S}\left[, i \in\left\{1, \ldots, 2^{N}-1\right\},\right. \\
\left.S_{0}^{N}=\right] x_{0}^{N}, x_{0}^{N}+\frac{1}{2} h_{N} \alpha_{S}\left[, S_{2^{N}}^{N}=\right] x_{2^{N}}^{N}-\frac{1}{2} h_{N} \alpha_{S}, x_{2^{N}}^{N}[, \\
\left.T_{i}^{N}=\right] x_{i}^{N}+\frac{1}{2} h_{N} \alpha_{S}, x_{i+1}^{N}-\frac{1}{2} h_{N} \alpha_{S}\left[, i \in\left\{0, \ldots, 2^{N}-1\right\}\right.
\end{gathered}
$$

where we denote by $\alpha_{S}$ the solid proportion. Each mass is connected to its neighbouring masses by springs of stiffness $k_{N}$. Moreover we denote by $\rho$ the mass density of the solid part that is supposed to be constant over ] $0, L\left[\right.$. Consequently the mass $m_{N}$ of any solid part $S_{i}^{N}$ is equal to $\rho \frac{\alpha_{S} L}{2^{N}}=\rho \alpha_{S} h_{N}$.

We assume without loss of generality that $L=1$. We denote by $u_{i}^{N}(t)$ the displacement of $x_{i}^{N}$ at time $t$. The coupling between the tree and the spring-mass system relies on the variables $p_{i}^{N}(t)$, which are the pressures 
at the outlets $i \in\left\{0, \ldots, 2^{N}-1\right\}$ and in the air pockets $T_{i}^{N}$. Each $T_{i}^{N}$ represents an alveolus, which is put in connection with the $i$-th outlet of the $N$-generation tree. Its "volume" (in fact length in this 1D model) is $\left|T_{i}^{n}\right|=x_{N+1}^{N}-x_{i}^{N}-\alpha_{S} h_{N}$, so that the flow rate which enters the tree at leaf $i$, which we shall denote by $q_{i}^{N}$, is the opposite of the volume derivative: $q_{i}^{N}=-\left(\dot{u}_{N+1}^{N}-\dot{u}_{i}^{N}\right)$ (the fluid is assumed incompressible). We set

$$
u_{0}^{N}(t)=u_{2^{N}}^{N}(t)=0 \quad \forall t \in[0, T] .
$$

Now consider $i$ between 1 and $2^{N}-1$. The pull-back force exerted by mass $i-1$ on $S_{i}^{N}$ is $-k_{N}\left(u_{i}^{N}(t)-u_{i-1}^{N}(t)\right)$. Similarly, the force exerted by mass $i+1$ on $S_{i}^{N}$ is $-k_{N}\left(u_{i}^{N}(t)-u_{i+1}^{N}(t)\right)$. The balance reads

$$
k_{N}\left(u_{i+1}^{N}(t)-2 u_{i}^{N}(t)+u_{i-1}^{N}(t)\right), \text { for } 1 \leq i \leq 2^{N}-1 .
$$

There is also a load on the $i$ th mass that is due to the fluid pressure on each side of $S_{i}^{N}$ :

$$
-p_{i}^{N}(t)+p_{i-1}^{N}(t), \text { for } 1 \leq i \leq 2^{N}-1
$$

So that the Newton's law for the $i$ th mass, $i \in\left\{1, \ldots, 2^{N}-1\right\}$, reads

$$
m_{N} \ddot{u}_{i}^{N}(t)-k_{N}\left(u_{i+1}^{N}(t)-2 u_{i}^{N}(t)+u_{i-1}^{N}(t)\right)+\left(p_{i}^{N}(t)-p_{i-1}^{N}(t)\right)=m_{N} f_{i}^{N}(t),
$$

where $f_{i}^{N}$ is a given exterior force applied on $S_{i}^{N}$. We recall that for all $i \in\left\{0, \ldots, 2^{N}-1\right\}$,

$$
p_{i}^{N}(t)=\sum_{j=0}^{2^{N}-1} R_{N-\nu_{i j}} q_{j}^{N}
$$

where $R_{k}=\sum_{l=0}^{k} r_{l}, \nu_{i j}$ is given by (2) and $q_{j}^{N}$ is the flux vector at the outlet of the tree and is given by:

$$
q_{j}^{N}(t)=-\left(\dot{u}_{j+1}^{N}(t)-\dot{u}_{j}^{N}(t)\right), j \in\left\{0, \ldots, 2^{N}-1\right\} .
$$

From the definition (3) of $A^{N} \in \mathcal{M}_{2^{N}}$, we deduce that for all $i \in\left\{1, \ldots, 2^{N}-1\right\}$

$$
p_{i}^{N}(t)-p_{i-1}^{N}(t)=\sum_{j=1}^{2^{N}} A_{i-1, j-1}^{N}\left(\dot{u}_{j}^{N}(t)-\dot{u}_{j-1}^{N}(t)\right)-\sum_{j=1}^{2^{N}} A_{i, j-1}^{N}\left(\dot{u}_{j}^{N}(t)-\dot{u}_{j-1}^{N}(t)\right) .
$$

Therefore the Newtonian relation of dynamic (12) satisfied by $u_{i}^{N}$ becomes, for $i \in\left\{1, \ldots, 2^{N}-1\right\}$

$$
\begin{array}{r}
m_{N} \ddot{u}_{i}^{N}(t)-k_{N}\left(u_{i+1}^{N}(t)-2 u_{i}^{N}(t)+u_{i-1}^{N}(t)\right) \\
+\sum_{j=1}^{2^{N}} A_{i-1, j-1}^{N}\left(\dot{u}_{j}^{N}(t)-\dot{u}_{j-1}^{N}(t)\right)-\sum_{j=1}^{2^{N}} A_{i, j-1}^{N}\left(\dot{u}_{j}^{N}(t)-\dot{u}_{j-1}^{N}(t)\right)=m_{N} f_{i}^{N}(t) .
\end{array}
$$

Additionnaly, let us be given $\left(u_{i, \alpha}^{N}\right)_{0 \leq i \leq 2^{N}} \in \mathbb{R}^{2^{N}+1}, \alpha \in\{0,1\}$, we consider the initial conditions

$$
u_{i}^{N}(0)=u_{i, 0}^{N}, \dot{u}_{i}^{N}(0)=u_{i, 1}^{N}, \text { for } 0 \leq i \leq 2^{N} .
$$

satisfying the compatibility conditions: $u_{0, \alpha}^{N}=u_{2^{N}, \alpha}^{N}=0$. 
Remark 2.1. The previous system of equations (15)-(16)-(11) is a system of ODE where, because of the tree, the displacement $u_{i}^{N}$ depends on all the other displacements. Thus the tree induces a non local, dissipative effect.

Remark 2.2. If we had assumed that the air in the alveoli could only escape through independent pipes (not connected to the other ones) then the matrix $A^{N}$ would have been diagonal and the system would have been:

$$
m_{N} \ddot{u}_{i}^{N}(t)-k_{N}\left(u_{i+1}^{N}(t)-2 u_{i}^{N}(t)+u_{i-1}^{N}(t)\right)-r_{N}\left(\dot{u}_{i+1}^{N}(t)-2 \dot{u}_{i}^{N}(t)+\dot{u}_{i-1}^{N}(t)\right)=m_{N} f_{i}^{N}(t)
$$

where $r_{N}$ is the resistance of a single pipe (assuming that all the pipes have the same resistance).

First we state a result of existence and uniqueness of the solution of (15)-(16)-(11).

Proposition 2.3. Assuming that $\left(f_{i}^{N}\right)_{1 \leq i \leq 2^{N-1}} \in\left(L^{2}(0, T)\right)^{2^{N}-1}$, then, there exists a unique solution $\left(u_{i}^{N}\right)_{0 \leq i \leq 2^{N}} \in\left(H^{2}(0, T)\right)^{2^{N}+1}$ of the problem defined by equations (15), boundary conditions (11) and initial conditions (16). Moreover the solution satisfies the following discrete energy estimate:

$$
\begin{aligned}
\frac{m_{N}}{2} \sum_{i=1}^{2^{N}-1}\left|\dot{u}_{i}^{N}(t)\right|^{2}+\frac{1}{2} k_{N} & \sum_{i=0}^{2^{N}-1}\left(u_{i+1}^{N}(t)-u_{i}^{N}(t)\right)^{2} \\
& \leq e^{t}\left(\frac{m_{N}}{2} \sum_{i=1}^{2^{N}-1}\left|\dot{u}_{i, 1}^{N}\right|^{2}+\frac{k_{N}}{2} \sum_{i=0}^{2^{N}-1}\left(u_{i+1,0}^{N}-u_{i, 0}^{N}\right)^{2}+\frac{m_{N}}{2} \sum_{i=1}^{2^{N}-1} \int_{0}^{t}\left|f_{i}^{N}(s)\right|^{2} \mathrm{~d} s\right)
\end{aligned}
$$

Proof. Since (15) is a system of ODE, the existence and uniqueness of a solution is standard. To obtain the discrete energy estimate we multiply the $i$ th equation of (15) by $\dot{u}_{i}^{N}$ and add all the contributions for $i=1, \ldots 2^{N}-1$. Next applying discrete integration by parts and remembering that $A^{N}$ is symmetric positive we can apply Gronwall Lemma which leads to (18). Detailed calculations will be provided in the proof of Lemma 1, which establishes a similar property at the continuous level.

\subsection{Reformulation}

In this subsection, we rewrite the system of ODE (15) as a PDE. This approach is based on an identification of arrays of discrete unknowns with functions defined on [0,1] (see [6], where a similar approach is applied to a set of $1 \mathrm{D}$ particles embedded in a lubricating fluid).

Let $u$ be the continuous function with respect to the space variable, affine in each cell for all $t$ and such that $u\left(x_{i}^{N}, t\right)=u_{i}^{N}(t)$ where $\left(u_{i}^{N}\right)_{0 \leq i \leq 2^{N}}$ is the solution of (11), (15), (16). First, we introduce the following functional spaces:

$$
\begin{aligned}
V_{N}= & \{v:[0,1] \rightarrow \mathbb{R} \text { s. t. } v \text { is a continuous function on }[0,1] \\
& \left.v \text { is an affine function on } C_{i}^{N} \text { for } i \in\left\{0, \ldots, 2^{N}-1\right\} \text { and } v(0)=v(1)=0\right\}
\end{aligned}
$$

and

$$
M_{N}=\{\mu:] 0,1[\rightarrow \mathbb{R}, \mu \text { is piecewise constant on }] 0,1\left[: \exists\left(\mu_{i}^{N}\right) \in \mathbb{R}^{2^{N}} \text { s. t. } \mu=\mu_{i}^{N} \text { on } C_{i}^{N}\right\}
$$

Note that $u_{N}(\cdot, t)$ is an element of $V_{N}$ and that $V_{N}$ is a close subspace of $H_{0}^{1}(0,1)$.

Let $v \in V_{N}$, on each cell $C_{i}^{N}, 0 \leq i \leq 2^{N}-1$, we have

$$
\partial_{x} v(x)=\frac{v\left(x_{i+1}^{N}\right)-v\left(x_{i}^{N}\right)}{h_{N}}, \quad x \in C_{i}^{N}
$$


hence it yields

$$
\partial_{x x} v(x)=\sum_{i=1}^{2^{N}-1} \frac{v\left(x_{i+1}^{N}\right)-2 v\left(x_{i}^{N}\right)+v\left(x_{i-1}^{N}\right)}{h_{N}} \delta_{x_{i}^{N}}(x) \quad \text { in } \mathcal{D}^{\prime}(0,1) .
$$

Similarly, let $\mu$ be given in $M_{N}$, we have

$$
\partial_{x} \mu(x)=\sum_{i=0}^{2^{N}-1}\left(\mu_{i+1}^{N}-\mu_{i}^{N}\right) \delta_{x_{i}^{N}}(x) \quad \text { in } \mathcal{D}^{\prime}(0,1) .
$$

Consequently considering $u^{N}$ and $p^{N}$ such that for all $\left.t \in\right] 0, T\left[, u^{N}(t, \cdot) \in V_{N}\right.$ with $u^{N}\left(t, x_{i}^{N}\right)=u_{i}^{N}(t)$ and $p^{N}(t, \cdot) \in M_{N}$ with $p^{N}(t, x)=p_{i}^{N}(t)$ for $x \in C_{i}^{N}$, the system of ODE (15) can be written as follows:

$$
m_{N} \sum_{i=1}^{2^{N}-1} \partial_{t t} u^{N}\left(x_{i}^{N}, t\right) \delta_{x_{i}^{N}}(x)-k_{N} h_{N} \partial_{x x} u^{N}(x, t)-\partial_{x} p^{N}(x, t)=m_{N} \sum_{i=1}^{2^{N}-1} f_{i}^{N}(t) \delta_{x_{i}^{N}}(x) .
$$

Furthermore, thanks to (13), (14) and the fact that

$$
q_{i}^{N}=\partial_{t} u^{N}\left(x_{i+1}^{N}, t\right)-\partial_{t} u^{N}\left(x_{i}^{N}, t\right)=\int_{C_{i}^{N}} \partial_{x} \partial_{t} u^{N}(y, t) \mathrm{d} y, \text { for all } 0 \leq i \leq 2^{N}-1,
$$

we find, using Proposition 1.5,

$$
p^{N}(x, t)=\int_{0}^{1} K_{N}(x, y) \partial_{x} \partial_{t} u^{N}(y, t) \mathrm{d} y
$$

hence

$$
\partial_{x} p^{N}(x, t)=\partial_{x} \partial_{t} \int_{0}^{1} K_{N}(x, y) \partial_{x} u^{N}(y, t) \mathrm{d} y
$$

Putting now (21) and (20) together, we obtain that $u^{N}$ satisfies the following PDE, $\forall t \in[0, T]$,

$$
\begin{aligned}
& m_{N} \sum_{i=1}^{2^{N}-1} \partial_{t t} u^{N}\left(x_{i}^{N}, t\right) \delta_{x_{i}^{N}}(x)-k_{N} h_{N} \partial_{x x} u^{N}(x, t) \\
& -\partial_{x} \partial_{t} \int_{0}^{1} K_{N}(x, y) \partial_{x} u^{N}(y, t) \mathrm{d} y=m_{N} \sum_{i=1}^{2^{N}-1} f_{i}^{N}(t) \delta_{x_{i}^{N}}(x), \text { in } \mathcal{D}^{\prime}(] 0,1[) .
\end{aligned}
$$

\subsection{The limit model}

In this section we study the behavior of $u^{N}$ as $N$ goes to infinity. We will prove that, under suitable assumptions on the data together with the "convergence" of the tree, the sequence $\left(u^{N}\right)_{N}$ converges (in a sense to be made precise) towards the weak solution $u$ of the following PDE:

$$
\rho \alpha_{S} \partial_{t t} u(x, t)-k \partial_{x x} u(x, t)-\partial_{t} \partial_{x} \int_{0}^{1} K(x, y) \partial_{y} u(y, t) \mathrm{d} y=\rho \alpha_{S} f(x, t),
$$

where $k$ is such that:

$$
k=k_{N} h_{N} .
$$

Note that the stiffness of each individual spring blows like $2^{N}$. It is natural, as those springs can be seen as small pieces of a given one-dimensional elastic material, and in this situation the stiffness of a portion, seen as a spring, is proportional to the reciprocal of its length. Another way to express this is to observe that the mechanical energy of the spring chain is bounded as $N$ goes to infinity. 
Note that the first two terms of equation (23) correspond to the wave equation that comes from the massspring chain and the third term is a viscous non-local term coming from the tree of pipes.

In order to pass to the limit in the discrete problem we assume that there exists $f \in L^{2}\left(0, T ; L^{2}(0,1)\right)$, $u_{1} \in L^{2}(0,1)$ and $u_{0} \in H_{0}^{1}(0,1)$ such that as $N$ goes to infinity:

$$
\begin{array}{r}
\sum_{i=1}^{2^{N}-1} f_{i}^{N}(t) \chi_{S_{i}^{N}}(x) \rightarrow \alpha_{S} f, \text { weakly in } L^{2}\left(0, T ; L^{2}(0,1)\right), \\
\sum_{i=1}^{2^{N}-1} u_{i, 1}^{N} \chi_{S_{i}^{N}}(x) \rightarrow \alpha_{S} u_{1} \text { weakly in } L^{2}(0,1), \\
u_{0}^{N} \rightarrow u_{0} \text { weakly in } H^{1}(0,1) .
\end{array}
$$

We may now state the main result of this section:

Theorem 2.4. Let us assume that (24), (25), (26), (27) hold true, and that $\sum_{n=0}^{N} \frac{r_{n}}{2^{n}}$ is convergent, then the sequence of solutions $u^{N}$ of (22) such that $u^{N}\left(0, x_{i}^{N}\right)=u_{0, i}^{N}$ and $\partial_{t} u^{N}\left(0, x_{i}^{N}\right)=u_{1, i}^{N}$ satisfies the following convergences as $N$ goes to infinity:

$$
u^{N} \rightarrow u \text { weakly } * \text { in } L^{\infty}\left(0, T ; H_{0}^{1}(0,1)\right),
$$

and

$$
\partial_{t} u^{N} \rightarrow \partial_{t} u \text { weakly } * \text { in } L^{\infty}\left(0, T ; L^{2}(0,1)\right),
$$

where $u$ is the unique weak solution of (23) satisfying $u(0)=u_{0}$ and $\partial_{t} u(0)=u_{1}$ in the following sense:

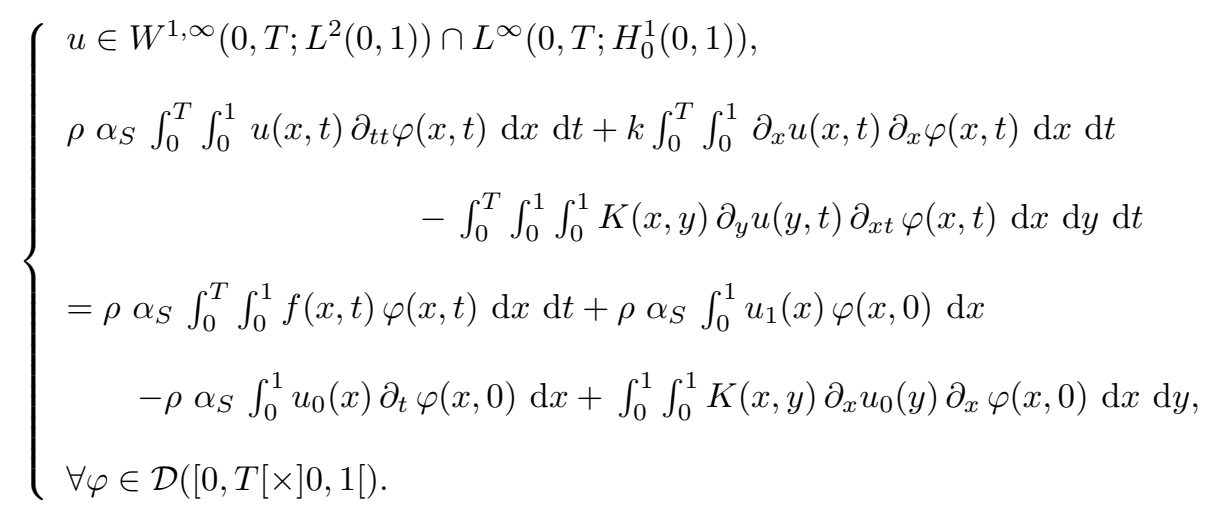

Proof. The proof is decomposed onto three steps.

Step 1 (energy estimates). First we derive energy estimates for $u^{N}$.

Lemma 1. Let us assume that conditions (24) and (25), (26), (27) are fullfilled, then ( $\left.u^{N}\right)$ is bounded in $L^{\infty}\left(0, T ; H_{0}^{1}(0,1)\right)$ and $\left(\partial_{t} u^{N}\right)$ is bounded in $L^{\infty}\left(0, T ; L^{2}(0,1)\right)$.

Proof. Multiplying $(22)$ by $\partial_{t} u^{N}(x, t)$ we obtain after integration over $] 0,1[$ :

$$
\begin{aligned}
& \frac{m_{N}}{2} \frac{\mathrm{d}}{\mathrm{d} t} \sum_{i=1}^{2^{N}-1}\left|\partial_{t} u^{N}\left(x_{i}^{N}, t\right)\right|^{2}+\frac{1}{2} k_{N} h_{N} \frac{\mathrm{d}}{\mathrm{d} t}\left\|\partial_{x} u^{N}(\cdot, t)\right\|_{L^{2}(0,1)}^{2} \\
& \quad+\int_{0}^{1} \int_{0}^{1} K_{N}(x, y) \partial_{x} \partial_{t} u^{N}(x, t) \partial_{x} \partial_{t} u^{N}(y, t) \mathrm{d} x \mathrm{~d} y=m_{N} \sum_{i=1}^{2^{N}-1} f_{i}^{N}(t) \partial_{t} u^{N}\left(x_{i}^{N}, t\right)
\end{aligned}
$$


Using that $\int_{0}^{1} \int_{0}^{1} K_{N}(x, y) \partial_{x} \partial_{t} u^{N}(x, t) \partial_{x} \partial_{t} u^{N}(y, t) \mathrm{d} x \mathrm{~d} y \geq 0$, and Young's inequality, we have

$$
\frac{m_{N}}{2} \frac{\mathrm{d}}{\mathrm{d} t} \sum_{i=1}^{2^{N}-1}\left|\partial_{t} u^{N}\left(x_{i}^{N}, t\right)\right|^{2}+\frac{1}{2} k_{N} h_{N} \frac{\mathrm{d}}{\mathrm{d} t}\left\|\partial_{x} u^{N}(\cdot, t)\right\|_{L^{2}(0,1)}^{2} \leq \frac{m_{N}}{2} \sum_{i=1}^{2^{N}-1}\left|\partial_{t} u^{N}\left(x_{i}^{N}, t\right)\right|^{2}+\frac{m_{N}}{2} \sum_{i=1}^{2^{N}-1}\left|f_{i}^{N}(t)\right|^{2} .
$$

Thus, recalling the Gronwall lemma we obtain

$$
\begin{aligned}
\frac{m_{N}}{2} \sum_{i=1}^{2^{N}-1}\left|\partial_{t} u^{N}\left(x_{i}^{N}, t\right)\right|^{2}+ & \frac{1}{2} k_{N} h_{N}\left\|\partial_{x} u^{N}(\cdot, t)\right\|_{L^{2}(0,1)}^{2} \\
& \leq e^{t}\left(\frac{m_{N}}{2} \sum_{i=1}^{2^{N}-1}\left|u_{i, 1}^{N}\right|^{2}+\frac{1}{2} k_{N} h_{N}\left\|\partial_{x} u_{0}^{N}\right\|_{L^{2}(0,1)}^{2}+\int_{0}^{t} \frac{m_{N}}{2} \sum_{i=1}^{2^{N}-1}\left|f_{i}^{N}(s)\right|^{2} \mathrm{~d} s\right)
\end{aligned}
$$

where $u_{0}^{N}$ belongs to $V_{N}$ and is such that $u_{0}^{N}\left(x_{i}^{N}\right)=u_{i, 0}^{N}$. We have assumed that $\left(\sum_{i=1}^{2^{N}-1} u_{i, 1}^{N} \chi_{i}^{N}\right)$ and $\left(u_{0}^{N}\right)$ converge weakly respectively in $L^{2}(0,1)$ and $H^{1}(0,1)$, thus noting that $\left\|\sum_{i=1}^{2^{N}-1} u_{i, 1}^{N} \chi_{i}^{N}\right\|_{L^{2}(0,1)}^{2}=h_{N} \sum_{i=1}^{2^{N}-1}\left|u_{i, 1}^{N}\right|^{2}$ we deduce that there exists a constant $C>0$ independent of $N$ such that

$$
\begin{aligned}
h_{N} \sum_{i=1}^{2^{N}-1}\left|u_{i, 1}^{N}\right|^{2} & \leq C, \\
\left\|\partial_{x} u_{0}^{N}\right\|_{L^{2}(0,1)}^{2} & \leq C .
\end{aligned}
$$

Moreover since $\left\|\sum_{i=1}^{2^{N}-1} f_{i}^{N}(t) \chi_{S_{i}^{N}}(x)\right\|_{L^{2}(0,1)}^{2}=\alpha_{S} h_{N} \sum_{i=1}^{2^{N}-1}\left|f_{i}^{N}(t)\right|^{2}$ and thanks to assumption (25) we have that

$$
h_{N} \int_{0}^{t} \sum_{i=1}^{2^{N}-1}\left|f_{i}^{N}(s)\right|^{2} \mathrm{~d} s \leq C
$$

Note that (31), (32), (33) imply the convergences (25), (26), (27) but only up to the extraction of subsequences. From estimate (30), together with (31), (32), (33) and under the assumption on the spring stiffness (24), we deduce that

$$
\left\|\partial_{x} u^{N}\right\|_{L^{\infty}\left(0, T ; L^{2}(0,1)\right)} \leq C
$$

Consequently, the first assertion holds true by Poincaré inequality, since $u^{N}(t) \in H_{0}^{1}(0,1)$, for almost every time $t$. Next thanks to (30), $m_{N} \sum_{i=1}^{2^{N}-1}\left|\partial_{t} u^{N}\left(x_{i}^{N}, t\right)\right|^{2}$ is bounded independently of $N$ and recalling that $m_{N}=\rho \alpha_{S} h_{N}$, we obtain that $\partial_{t} u^{N}$ is bounded in $L^{\infty}\left(0, T ; L^{2}(0,1)\right)$, independently of $N$. Indeed we have

$$
\partial_{t} u^{N}=\dot{u}_{i}^{N}+\frac{\left(x-x_{i}^{N}\right)}{h_{N}}\left(\dot{u}_{i+1}^{N}-\dot{u}_{i}^{N}\right), \text { on } C_{i}^{N}
$$


therefore it is easy to see that

$$
\begin{aligned}
\left\|\partial_{t} u^{N}(t)\right\|_{L^{2}(0,1)}^{2} & =\sum_{i=0}^{2^{N}-1} \int_{C_{i}^{N}}\left(\dot{u}_{i}^{N}+\frac{\left(x-x_{i}^{N}\right)}{h_{N}}\left(\dot{u}_{i+1}^{N}-\dot{u}_{i}^{N}\right)\right)^{2} \mathrm{~d} x \\
& \leq C h_{N} \sum_{i=1}^{2^{N}-1}\left|\dot{u}_{i}^{N}\right|^{2}, \quad \text { since } u_{0,1}^{N}=u_{2^{N}, 1}^{N}=0 \text { and }\left|C_{i}^{N}\right|=h_{N}, \\
& \leq C m_{N} \sum_{i=1}^{2^{N}-1}\left|\dot{u}_{i}^{N}\right|^{2}, \quad \text { since } m_{N}=\rho \alpha_{S} h_{N} .
\end{aligned}
$$

Remark 2.5. From energy equality (29) and under assumptions (26), (27), (25) we can deduce that there exists $C$ independent of $N$ such that

$$
0 \leq \int_{0}^{T} \int_{0}^{1} \int_{0}^{1} K_{N}(x, y) \partial_{t} \partial_{x} u^{N}(x, t) \partial_{t} \partial_{x} u^{N}(y, t) \mathrm{d} x \mathrm{~d} y \mathrm{~d} t \leq C .
$$

From the previous boundedness condition we are able to prove additional bounds in some special cases. In particular when $r_{n}=r_{0} \alpha^{n}$ and $1<\alpha<2$, thanks to Proposition 1.19 we have that $\partial_{x} \partial_{t} u^{N}$ is bounded independently of $N$ in $\left.L^{2}\left(0, T ; H^{s}(0,1)\right)\right), s=\frac{\ln (\alpha)}{2 \ln (2)}-\frac{1}{2}$ thus $\partial_{t} u^{N}$ is bounded independently of $N$ in $\left.L^{2}\left(0, T ; H^{s+1}(0,1)\right)\right)$, $s+1=\frac{\ln (\alpha)}{2 \ln (2)}+\frac{1}{2}$.

Step 2 (passage to the limit). Lemma 1 leads to the following weak convergences: up to a subsequence still denoted by $u^{N}$, we have that there exists $u \in W^{1, \infty}\left(0, T ; L^{2}(0,1)\right) \cap L^{\infty}\left(0, T ; H_{0}^{1}(0,1)\right)$, such that

$$
u^{N} \rightarrow u \text { weakly } * \text { in } L^{\infty}\left(0, T ; H_{0}^{1}(0,1)\right),
$$

and

$$
\partial_{t} u^{N} \rightarrow \partial_{t} u \text { weakly } * \text { in } L^{\infty}\left(0, T ; L^{2}(0,1)\right) .
$$

It remains to prove that $u$ satisfies the weak formulation $(28)$. Let $\varphi$ be in $H_{0}^{1}(0,1)$ and $\psi$ in $\mathcal{D}([0, T))$. Multiplying (22) by $\varphi(x) \psi(t)$ and integrating in time and space and after integrating by parts, equation (22) becomes

$$
\begin{gathered}
\rho \alpha_{S} h_{N} \int_{0}^{T} \sum_{i=1}^{2^{N}-1} u^{N}\left(x_{i}^{N}, t\right) \varphi\left(x_{i}^{N}\right) \ddot{\psi}(t) \mathrm{d} t+k \int_{0}^{T} \int_{0}^{1} \partial_{x} u^{N}(x, t) \partial_{x} \varphi(x) \psi(t) \mathrm{d} x \mathrm{~d} t \\
-\int_{0}^{T} \int_{0}^{1} \int_{0}^{1} K_{N}(x, y) \partial_{x} u^{N}(y, t) \partial_{x} \varphi(x) \dot{\psi}(t) \mathrm{d} x \mathrm{~d} y \mathrm{~d} t \\
=\rho \alpha_{S} h_{N} \int_{0}^{T} \sum_{i=1}^{2^{N}-1} f_{i}^{N}(t) \varphi\left(x_{i}^{N}\right) \psi(t) \mathrm{d} t+\rho \alpha_{S} h_{N} \sum_{i=1}^{2^{N}-1} u_{i, 1}^{N} \varphi\left(x_{i}^{N}\right) \psi(0) \\
-\rho \alpha_{S} h_{N} \sum_{i=1}^{2^{N}-1} u_{i, 0}^{N} \varphi\left(x_{i}^{N}\right) \dot{\psi}(0)+\int_{0}^{1} \int_{0}^{1} K_{N}(x, y) \partial_{x} u_{0}^{N}(y) \partial_{x} \varphi(x) \psi(0) \mathrm{d} x \mathrm{~d} y .
\end{gathered}
$$

Let us begin with the second left-hand side term. As $u^{N} \rightarrow u$ weakly $*$ in $L^{\infty}\left(0, T ; H^{1}(0,1)\right)$, as $N \rightarrow+\infty$, we have

$$
\int_{0}^{T} \int_{0}^{1} \partial_{x} u^{N}(x, t) \partial_{x} \varphi(x) \psi(t) \mathrm{d} x \mathrm{~d} t \rightarrow \int_{0}^{T} \int_{0}^{1} \partial_{x} u(x, t) \partial_{x} \varphi(x) \psi(t) \mathrm{d} x \mathrm{~d} t .
$$


Next, we consider the third left-hand side term. Referring to Theorem 1.7 and under the assumption that the sequence $\sum_{n=0}^{N} \frac{r_{n}}{2^{n}}$ is convergent, we know that $K_{N}(x, y) \rightarrow K(x, y)$ strongly in $L^{1}((0,1) \times(0,1))$, as $N$ tends to infinity. Furthermore, since $\partial_{x} u^{N}$ is bounded in $L^{2}\left(0, T ; L^{2}(0,1)\right)$, and

$$
\left\|\mathcal{R}_{N} \partial_{x} u^{N}(\cdot, t)-\mathcal{R} \partial_{x} u^{N}(\cdot, t)\right\|_{L^{2}(0,1)} \leq\left\|K_{N}-K\right\|_{L^{1}(] 0,1[\times] 0,1[)}\left\|\partial_{x} u^{N}(\cdot, t)\right\|_{L^{2}(0,1)}, \text { a.e. in } t
$$

we deduce that $\mathcal{R}_{N} \partial_{x} u^{N}-\mathcal{R} \partial_{x} u^{N}$ tends to zero in $L^{2}\left(0, T ; L^{2}(0,1)\right)$ as $N$ goes to infinity. Therefore from the continuity of $\mathcal{R}$ on $L^{2}(0,1)$ we obtain that $\left(\mathcal{R} \partial_{x} u^{N}\right)_{N}$ (up to the extraction of a subsequence) weakly converges to $\mathcal{R} \partial_{x} u$ in $L^{2}\left(0, T ; L^{2}(0,1)\right)$ and thus:

$$
\mathcal{R}_{N} \partial_{x} u^{N} \rightarrow \mathcal{R} \partial_{x} u \text { weakly in } L^{2}\left(0, T ; L^{2}(0,1)\right) \text {, when } N \rightarrow+\infty \text {. }
$$

Consequently, we have, when $N$ goes to infinity,

$$
\int_{0}^{T}\left(\mathcal{R}_{N} \partial_{x} u^{N}, \partial_{x} \varphi \dot{\psi}\right) \mathrm{d} t \longrightarrow \int_{0}^{T}\left(\mathcal{R} \partial_{x} u, \partial_{x} \varphi \dot{\psi}\right) \mathrm{d} t
$$

Remark 2.6. In the previous convergence we did not use the fact that $\mathcal{R}$ is a compact operator in $L^{2}$. The compactness of $\mathcal{R}$ implies that the convergence (36) is strong.

Next we consider the inertial term for which we will prove the following lemma:

Lemma 2. We have

$$
\lim _{N \rightarrow+\infty} \rho \alpha_{S} h_{N} \int_{0}^{T} \sum_{i=1}^{2^{N}-1} u^{N}\left(x_{i}^{N}, t\right) \varphi\left(x_{i}^{N}\right) \ddot{\psi}(t) \mathrm{d} t=\rho \alpha_{S} \int_{0}^{T} \int_{0}^{1} u(x, t) \varphi(x) \ddot{\psi}(t) \mathrm{d} x \mathrm{~d} t
$$

for all $\varphi \in H_{0}^{1}(0,1)$ and $\psi \in \mathcal{D}([0, T[)$.

Proof. Let us introduce $D_{N}$ defined as follows

$$
D_{N}=\alpha_{S} h_{N} \int_{0}^{T} \sum_{i=1}^{2^{N}-1} u^{N}\left(x_{i}^{N}, t\right) \varphi\left(x_{i}^{N}\right) \ddot{\psi}(t) \mathrm{d} t-\int_{0}^{T} \int_{0}^{1} u^{N}(x, t) \varphi(x) \ddot{\psi}(t) \chi_{S}^{N}(x) \mathrm{d} x \mathrm{~d} t
$$

where $\chi_{S}^{N}$ is the characteristic function of the solid part on $] 0,1\left[\right.$. We wish to prove that $\lim _{N \rightarrow+\infty} D_{N}=0$. Thanks to the boundary conditions we have:

$$
D_{N}=\alpha_{S} h_{N} \int_{0}^{T} \sum_{i=0}^{2^{N}} u^{N}\left(x_{i}^{N}, t\right) \varphi\left(x_{i}^{N}\right) \ddot{\psi}(t) \mathrm{d} t-\int_{0}^{T} \int_{0}^{1} u^{N}(x, t) \varphi(x) \ddot{\psi}(t) \chi_{S}^{N}(x) \mathrm{d} x \mathrm{~d} t
$$

Using next that $\left|S_{i}^{N}\right|=\alpha_{S} h_{N}$, we immediately see that

$$
D_{N}=\int_{0}^{T} \int_{0}^{1} \sum_{i=0}^{2^{N}}\left(u^{N}\left(x_{i}^{N}, t\right) \varphi\left(x_{i}^{N}\right)-u^{N}(x, t) \varphi(x)\right) \chi_{S_{i}^{N}}^{N}(x) \ddot{\psi}(t) \mathrm{d} x \mathrm{~d} t
$$


where $\chi_{S_{i}^{N}}^{N}$ is the characteristic function corresponding to the solid part $S_{i}^{N}$. Furthermore, writing $u^{N}\left(x_{i}^{N}, t\right) \varphi\left(x_{i}^{N}\right)-$ $u^{N}(x, t) \varphi(x)$ as $u^{N}\left(x_{i}^{N}, t\right)\left(\varphi\left(x_{i}^{N}\right)-\varphi(x)\right)+\left(u^{N}\left(x_{i}^{N}, t\right)-u^{N}(x, t)\right) \varphi(x)$, we deduce that

$$
\begin{aligned}
\left|D_{N}\right| \leq & \int_{0}^{T} \int_{0}^{1} \sum_{i=0}^{2^{N}}\left|u^{N}\left(x_{i}^{N}, t\right) \| \varphi\left(x_{i}^{N}\right)-\varphi(x)\right| \chi_{S_{i}^{N}}^{N}(x)|\ddot{\psi}(t)| \mathrm{d} x \mathrm{~d} t \\
& +\int_{0}^{T} \int_{0}^{1} \sum_{i=0}^{2^{N}}\left|u^{N}\left(x_{i}^{N}, t\right)-u^{N}(x, t)\right||\varphi(x)| \chi_{S_{i}^{N}}^{N}(x)|\ddot{\psi}(t)| \mathrm{d} x \mathrm{~d} t .
\end{aligned}
$$

Let us now study the two right-hand side terms of (37) separately. First, we notice that

$$
\left|\varphi\left(x_{i}^{N}\right)-\varphi(x)\right|=\left|\int_{x}^{x_{i}^{N}} \partial_{x} \varphi(y) \mathrm{d} y\right| \leq\left|x-x_{i}^{N}\right|^{1 / 2}\left\|\partial_{x} \varphi(y)\right\|_{L^{2}(0,1)} \leq C\left|x-x_{i}^{N}\right|^{1 / 2} .
$$

Moreover, since $u^{N} \in L^{\infty}\left(0, T ; H_{0}^{1}(0,1)\right)$, and since we know that $H^{1}(0,1)$ is continuously embedded in $C^{0}([0,1])$, hence

$$
\left.\left|u^{N}\left(x_{i}^{N}, t\right)\right| \leq C \text { a.e. } t \in\right] 0, T\left[\text { and for all } 0 \leq i \leq 2^{N} .\right.
$$

Putting now (38) and (39) together, we obtain

$$
\begin{aligned}
\int_{0}^{T} \int_{0}^{1} \sum_{i=0}^{2^{N}}\left|u^{N}\left(x_{i}^{N}, t\right)\right|\left|\varphi\left(x_{i}^{N}\right)-\varphi(x)\right| \chi_{S_{i}^{N}}^{N}(x)|\ddot{\psi}(t)| \mathrm{d} x \mathrm{~d} t & \\
& \leq C \sum_{i=0}^{2^{N}} \int_{S_{i}^{N}}\left|x-x_{i}^{N}\right|^{1 / 2} \mathrm{~d} x \leq C \sum_{i=0}^{2^{N}}\left(h_{N}\right)^{3 / 2} \leq C\left(h_{N}\right)^{1 / 2},
\end{aligned}
$$

hence,

$$
\lim _{N \rightarrow+\infty} \int_{0}^{T} \int_{0}^{1} \sum_{i=0}^{2^{N}}\left|u^{N}\left(x_{i}^{N}, t\right)\right|\left|\varphi\left(x_{i}^{N}\right)-\varphi(x)\right| \chi_{S_{i}^{N}}^{N}(x)|\ddot{\psi}(t)| \mathrm{d} x \mathrm{~d} t=0 .
$$

Similarly, since $u^{N}$ is bounded in $L^{\infty}\left(0, T ; H_{0}^{1}(0,1)\right)$ independently of $N$, we have

$$
\lim _{N \rightarrow+\infty} \int_{0}^{T} \int_{0}^{1} \sum_{i=0}^{2^{N}}\left|u^{N}\left(x_{i}^{N}, t\right)-u^{N}(x, t)\right||\varphi(x)| \chi_{S_{i}^{N}}^{N}(x)|\ddot{\psi}(t)| \mathrm{d} x \mathrm{~d} t=0 .
$$

Furthermore the sequence $\left(u^{N}\right)_{N}$ is bounded in $W^{1, \infty}\left(0, T ; L^{2}(0,1)\right) \cap L^{\infty}\left(0, T ; H_{0}^{1}(0,1)\right)$ which implies in particular that $\left(u^{N}\right)_{N}$ is bounded in $H^{1}(] 0, T[\times] 0,1[)$ and consequently is relatively compact in $L^{2}(] 0, T[\times] 0,1[)$. Moreover, we know that $\chi_{S}^{N} \rightarrow \alpha_{S}$ weakly $*$ in $L^{\infty}(0,1)$. Hence

$$
\int_{0}^{T} \int_{0}^{1} u^{N}(x, t) \varphi(x) \ddot{\psi}(t) \chi_{S}^{N}(x) \mathrm{d} x \mathrm{~d} t \rightarrow \alpha_{S} \int_{0}^{T} \int_{0}^{1} u(x, t) \varphi(x) \ddot{\psi}(t) \mathrm{d} x \mathrm{~d} t .
$$

The proof of Lemma 2 then follows immediately. 
Next we want to pass to the limit in the load term. To do this we need assumptions on the exterior load. We study the following difference

$$
\begin{aligned}
D_{N}^{1} & =\alpha_{S} h_{N} \int_{0}^{T} \sum_{i=1}^{2^{N}-1} f_{i}^{N}(t) \varphi\left(x_{i}^{N}\right) \psi(t) \mathrm{d} t-\int_{0}^{T} \int_{0}^{1} \sum_{i=1}^{2^{N}-1} f_{i}^{N}(t) \varphi(x) \chi_{S_{i}^{N}}(x) \psi(t) \mathrm{d} x \mathrm{~d} t \\
& =\int_{0}^{T} \sum_{i=1}^{2^{N}-1} f_{i}^{N}(t)\left(\int_{S_{i}^{N}}\left(\varphi\left(x_{i}^{N}\right)-\varphi(x)\right) \mathrm{d} x\right) \psi(t) \mathrm{d} t .
\end{aligned}
$$

Using (38) we obtain

$$
\begin{aligned}
\left|D_{N}^{1}\right| & \leq C \int_{0}^{T} \sum_{i=1}^{2^{N}-1}\left(h_{N}\right)^{3 / 2}\left|f_{i}^{N}(t)\right| \mathrm{d} t \\
& \leq C_{T}\left(h_{N}\right)^{1 / 2}\left(\int_{0}^{T} \sum_{i=1}^{2^{N}-1} h_{N}\left|f_{i}^{N}(t)\right|^{2}\right)^{1 / 2} \mathrm{~d} t
\end{aligned}
$$

Remembering assumption (33) on $f_{i}^{N}$, we deduce that $D_{N}^{1}$ goes to zero as $N$ goes to infinity.

Moreover, under the assumption that there exists $f \in L^{2}\left(0, T ; L^{2}(0,1)\right)$ such that $\sum_{i=1}^{2^{N}-1} f_{i}^{N}(t) \chi_{i}^{N}(x)$ converges weakly in $L^{2}\left(0, T ; L^{2}(0,1)\right)$ towards $\alpha_{S} f$ we have

$$
\int_{0}^{T} \int_{0}^{1} \sum_{i=1}^{2^{N}-1} f_{i}^{N}(t) \varphi(x) \chi_{S_{i}^{N}}(x) \psi(t) \mathrm{d} x \mathrm{~d} t \longrightarrow \alpha_{S} \int_{0}^{T} \int_{0}^{1} f(x, t) \varphi(x) \psi(t) \mathrm{d} x \mathrm{~d} t
$$

thus

$$
\alpha_{S} h_{N} \int_{0}^{T} \sum_{i=1}^{2^{N}-1} f_{i}^{N}(t) \varphi\left(x_{i}^{N}\right) \psi(t) \mathrm{d} t \longrightarrow \alpha_{S} \int_{0}^{T} \int_{0}^{1} f(x, t) \varphi(x) \psi(t) \mathrm{d} x \mathrm{~d} t .
$$

Now we take care of the terms containing the initial conditions. Under assumption (26) and repeating the argument used for the load term, we deduce that as $N$ goes to infinity:

$$
\alpha_{S} h_{N} \sum_{i=1}^{2^{N}-1} u_{i, 1}^{N} \varphi\left(x_{i}^{N}\right) \longrightarrow \alpha_{S} \int_{0}^{1} u_{1}(x) \varphi(x) \mathrm{d} x
$$

Next, as in the proof of Lemma 2, we have

$$
\lim _{N \rightarrow+\infty} h_{N} \sum_{i=1}^{2^{N}-1} u_{0}^{N}\left(x_{i}^{N}\right) \varphi\left(x_{i}^{N}\right)=\int_{0}^{1} u_{0}(x) \varphi(x) \mathrm{d} x
$$

Finally as for (36) we have

$$
\mathcal{R}_{N} \partial_{x} u_{0}^{N} \rightarrow \mathcal{R} \partial_{x} u_{0} \text { weakly in } L^{2}(0,1),
$$

which yields

$$
\int_{0}^{1} \int_{0}^{1} K_{N}(x, y) \partial_{x} u_{0}^{N}(y) \partial_{x} \varphi(x) \mathrm{d} x \mathrm{~d} y \longrightarrow \int_{0}^{1} K(x, y) \partial_{x} u_{0}(y) \partial_{x} \varphi(x) \mathrm{d} x \mathrm{~d} y .
$$

Consequently $u$ is a solution of (28).

Step 3 (uniqueness of the solution of (28)). It remains to prove the uniqueness of the solution of equation (28). As a consequence the whole sequence $\left(u^{N}\right)_{N}$ will converge towards $u$. 
Let $u_{1}, u_{2} \in W^{1, \infty}\left(0, T ; L^{2}(0,1)\right) \cap L^{\infty}\left(0, T ; H_{0}^{1}(0,1)\right)$ be two solutions of $(28)$ corresponding to the same data, then the function $u=u_{1}-u_{2}$ is a solution to the following problem:

$$
\left\{\begin{array}{l}
\rho \alpha_{S} \int_{0}^{T} \int_{0}^{1} u(x, t) \partial_{t t} \varphi(x, t) \mathrm{d} x \mathrm{~d} t+k \int_{0}^{T} \int_{0}^{1} \partial_{x} u(x, t) \partial_{x} \varphi(x, t) \mathrm{d} x \mathrm{~d} t \\
-\int_{0}^{T} \int_{0}^{1} \int_{0}^{1} K(x, y) \partial_{x} u(y, t) \partial_{x t} \varphi(x, t) \mathrm{d} x \mathrm{~d} y \mathrm{~d} t=0 \\
\forall \varphi \in \mathcal{D}([0, T[\times] 0,1[) .
\end{array}\right.
$$

Since $u \in W^{1, \infty}\left(0, T ; L^{2}(0,1)\right) \cap L^{\infty}\left(0, T ; H^{1}(0,1)\right)$ the trace of $u$ at time $t=0$ makes sense at least in $L^{2}(0,1)$. It is easy to verify that $u$ satisfies $u(0)=0$. Next we rewrite the term $\int_{0}^{T} \int_{0}^{1} u(x, t) \partial_{t t} \varphi(x, t) \mathrm{d} x \mathrm{~d} t$. After integration by parts with respect to the time and since $u(0)=0$ and $\partial_{t} \varphi(T)=0$ we obtain that

$$
\int_{0}^{T} \int_{0}^{1} u(x, t) \partial_{t t} \varphi(x, t) \mathrm{d} x \mathrm{~d} t=-\int_{0}^{T} \int_{0}^{1} \partial_{t} u(x, t) \partial_{t} \varphi(x, t) \mathrm{d} x \mathrm{~d} t
$$

therefore

$$
\left\{\begin{array}{l}
-\rho \alpha_{S} \int_{0}^{T} \int_{0}^{1} \partial_{t} u(x, t) \partial_{t} \varphi(x, t) \mathrm{d} x \mathrm{~d} t+k \int_{0}^{T} \int_{0}^{1} \partial_{x} u(x, t) \partial_{x} \varphi(x, t) \mathrm{d} x \mathrm{~d} t \\
-\int_{0}^{T} \int_{0}^{1} \int_{0}^{1} K(x, y) \partial_{x} u(y, t) \partial_{x t} \varphi(x, t) \mathrm{d} x \mathrm{~d} y \mathrm{~d} t=0, \\
\forall \varphi \in \mathcal{D}([0, T[\times] 0,1[) .
\end{array}\right.
$$

By a density argument this weak formulation (41) is also valid for all $\varphi \in H^{1}\left(0, T ; H_{0}^{1}(0,1)\right)$ with $\varphi(T)=0$. As for the wave equation, it is not possible to take $\varphi=u$ as a test function, since we do not have that $\partial_{x t} u$ in $L^{2}\left(0, T ; L^{2}(0,1)\right)$. Therefore, for any fixed $\left.s \in\right] 0, T[$, we set, as in $[7]$

$$
w(x, t)=\left\{\begin{array}{cc}
-\int_{t}^{s} u(x, \tau) \mathrm{d} \tau & \text { if } t<s \\
0 & \text { if } t \geq s
\end{array}\right.
$$

Now we can take $\varphi=w$ as a test function in the variational formulation (41), therefore,

$$
\begin{array}{r}
-\rho \alpha_{S} \int_{0}^{T} \int_{0}^{1} \partial_{t} u(x, t) \partial_{t} w(x, t) \mathrm{d} x \mathrm{~d} t+k \int_{0}^{T} \int_{0}^{1} \partial_{x} u(x, t) \partial_{x} w(x, t) \mathrm{d} x \mathrm{~d} t \\
-\int_{0}^{T} \int_{0}^{1} \int_{0}^{1} K(x, y) \partial_{x} u(y, t) \partial_{x t} w(x, t) \mathrm{d} x \mathrm{~d} y \mathrm{~d} t=0 .
\end{array}
$$

Recalling the definition (42) of $w$, it follows that

$$
\begin{array}{r}
-\frac{\rho \alpha_{S}}{2} \int_{0}^{s} \frac{\mathrm{d}}{\mathrm{d} t} \int_{0}^{1} u^{2}(x, t) \mathrm{d} x \mathrm{~d} t+\frac{k}{2} \int_{0}^{s} \frac{\mathrm{d}}{\mathrm{d} t} \int_{0}^{1}\left(\partial_{x} w(x, t)\right)^{2} \mathrm{~d} x \mathrm{~d} t \\
-\int_{0}^{s} \int_{0}^{1} \int_{0}^{1} K(x, y) \partial_{x} u(x, t) \partial_{x} u(y, t) \mathrm{d} x \mathrm{~d} y \mathrm{~d} t=0
\end{array}
$$

consequently, using $u(0)=0$ and $\partial_{t} w(\cdot, s)=0$, we deduce that

$$
-\frac{\rho \alpha_{S}}{2}\|u(\cdot, s)\|_{L^{2}(0,1)}^{2}-\frac{k}{2}\left\|\partial_{x} w(\cdot, 0)\right\|_{L^{2}(0,1)}^{2}=\int_{0}^{s} \int_{0}^{1} \int_{0}^{1} K(x, y) \partial_{x} u(y, t) \partial_{x} u(x, t) \mathrm{d} x \mathrm{~d} y \mathrm{~d} t \geq 0 .
$$


Inequality (43) means that

$$
\frac{\rho \alpha_{S}}{2}\|u(\cdot, s)\|_{L^{2}(0,1)}^{2}+\frac{k}{2}\left\|\partial_{x} w(\cdot, 0)\right\|_{L^{2}(0,1)}^{2} \leq 0
$$

hence $u(\cdot, s)=0$ a.e. on $] 0,1[$. Since $s$ is arbitrary in $] 0, T[$, we obtain that $u=0$ a.e. on $] 0,1[\times] 0, T[$.

This ends the proof of Theorem 2.4.

Remark 2.7. In the case where the air can escape thought $2^{N}$ non-connected pipes whose resistances $r_{N}$ are assumed to be equal to $\frac{r}{2^{N}}$ it is easy to prove that the limit equation corresponding to (17) is the standard strongly damped wave equation:

$$
\rho \alpha_{S} \partial_{t t} u-k \partial_{x x} u-r \partial_{x x} \partial_{t} u=\rho \alpha_{S} f .
$$

\subsubsection{Remarks on the assumptions made on the data}

In this subsection we build data that verify the assumptions $(25),(26),(27)$. Let $f$ be given in $L^{2}\left(0, T ; L^{2}(0,1)\right)$. We consider $f_{i}^{N}(t)$ defined by

$$
f_{i}^{N}(t)=\frac{1}{\alpha_{S} h_{N}} \int_{S_{i}^{N}} f(x, t) \mathrm{d} x, \quad 1 \leq i \leq 2^{N}-1
$$

Let us denote by $D_{N}^{2}$ the difference

$$
D_{N}^{2}=\int_{0}^{T} \int_{0}^{1}\left(\sum_{i=1}^{2^{N}-1} f_{i}^{N}(t) \chi_{S_{i}^{N}}(x)-f(x, t) \chi_{S}^{N}(x)\right) \varphi(x, t) \mathrm{d} x \mathrm{~d} t
$$

for $\varphi \in \mathcal{D}(] 0, T[\times] 0,1[)$. Since $\varphi$ has a compact support in $] 0, T[\times] 0,1\left[, D_{N}^{2}\right.$ can be written for $N$ sufficiently large:

$$
\begin{aligned}
D_{N}^{2} & =\int_{0}^{T} \int_{0}^{1}\left(\sum_{i=1}^{2^{N}-1} f_{i}^{N}(t) \chi_{S_{i}^{N}}(x)-f(x, t) \chi_{S_{i}^{N}}(x)\right) \varphi(x, t) \mathrm{d} x \mathrm{~d} t \\
& =\int_{0}^{T}\left(\sum_{i=1}^{2^{N}-1} \frac{1}{\alpha_{S} h_{N}}\left(\int_{S_{i}^{N}} f(x, t) \mathrm{d} x\right)\left(\int_{S_{i}^{N}} \varphi(x, t) \mathrm{d} x\right)-\int_{S_{i}^{N}} f(x, t) \varphi(x, t) \mathrm{d} x\right) \mathrm{d} t \\
& =\int_{0}^{T} \sum_{i=1}^{2^{N}-1} \int_{S_{i}^{N}} f(y, t)\left(\frac{1}{\alpha_{S} h_{N}} \int_{S_{i}^{N}} \varphi(x, t) \mathrm{d} x-\varphi(y, t)\right) \mathrm{d} y \mathrm{~d} t .
\end{aligned}
$$

Let $y$ be given in $S_{i}^{N}$, we have

$$
\frac{1}{\alpha_{S} h_{N}} \int_{S_{i}^{N}} \varphi(x, t) \mathrm{d} x-\varphi(y, t)=\frac{1}{\alpha_{S} h_{N}} \int_{S_{i}^{N}}(\varphi(x, t)-\varphi(y, t)) \mathrm{d} x .
$$

Since $\varphi \in \mathcal{D}(] 0, T[\times] 0,1[)$ the difference $|\varphi(x, t)-\varphi(y, t)|$ can be estimated by $C|x-y|$. Thus a simple calculation gives

$$
\left|\frac{1}{\alpha_{S} h_{N}} \int_{S_{i}^{N}} \varphi(x, t) \mathrm{d} x-\varphi(y, t)\right| \leq C h_{N} .
$$

Consequently $\left|D_{N}^{2}\right|$ can be estimated by $C h_{N}$ and tends to zero as $N$ goes to infinity. Moreover since

$$
\int_{0}^{T} \int_{0}^{1} f(x, t) \varphi(x, t) \chi_{S}^{N}(x) \mathrm{d} x \mathrm{~d} t \longrightarrow \alpha_{S} \int_{0}^{T} \int_{0}^{1} f(x, t) \varphi(x, t) \mathrm{d} x \mathrm{~d} t,
$$


we deduce that $\sum_{i=1}^{2^{N}-1} f_{i}^{N}(t) \chi_{S_{i}^{N}}(x)$ converges towards $\alpha_{S} f$ in $\mathcal{D}^{\prime}(] 0, T[\times] 0,1[)$. But thanks to the choice of $f_{i}^{N}$ we know that $\sum_{i=1}^{2^{N}-1} f_{i}^{N}(t) \chi_{S_{i}^{N}}(x)$ is bounded independently of $N$ in $L^{2}\left(0, T ; L^{2}(0,1)\right)$ thus the convergence of $\sum_{i=1}^{2^{N}-1} f_{i}^{N}(t) \chi_{S_{i}^{N}}(x)$ towards $\alpha_{S} f$ in $\mathcal{D}^{\prime}(] 0, T[\times] 0,1[)$ takes place in $L^{2}\left(0, T ; L^{2}(0,1)\right)$ endowed with its weak topology. The definition (44) implies that the assumption (25) is satisfied.

In order to satisfy $(26)$ we define for any $u_{1} \in L^{2}(0,1)$

$$
u_{i, 1}^{N}=\frac{1}{\alpha_{S} h_{N}} \int_{S_{i}^{N}} u_{1}(x) \mathrm{d} x, \quad 1 \leq i \leq 2^{N} .
$$

Finally it remains to satisfy (27), to do so, we consider for any $u_{0} \in H_{0}^{1}(0,1)$, its $H^{1}$ projection, $u_{0}^{N}$, on the finite dimensional space $V_{N}$.

\subsection{The case of a non convergent tree}

In this section we consider the case where $\sum \frac{r_{n}}{2^{n}}$ diverges. This is the case when $r_{n}=r_{0} \alpha^{n}$ with $\alpha \geq 2$. The case $\alpha=2$ corresponds to the optimal lung in the sense introduced by [10]. We will see that in this case when the number of generations of the tree of pipes tends to infinity, under the same hypothesis on the data we made previously, the solution of our one dimensional model converges towards the initial data $u_{0}$. This somewhat surprising behaviour is a consequence of the fact that, as pointed out in [10], the "optimal" value $\alpha$ (which is strictly greater than the measured value 1.63) leads to a tree which is divergent from the resistance point of view: it is impossible to have fluid circulate through it with finite energy. As motion is made possible by circulation through the tree only, everything stays at rest.

First we begin by a remark on the energy estimates that give additional bounds in some cases of non convergent tree of pipes. The energy estimates obtained in the discrete model are still valid and imply in particular that $\int_{0}^{T} \int_{0}^{1} \int_{0}^{1} K_{N}(x, y) \partial_{x} \partial_{t} u^{N}(x, t) \partial_{x} \partial_{t} u^{N}(y, t) \mathrm{d} x \mathrm{~d} y \mathrm{~d} t$ is bounded independently of $N$. But this term can be written:

$$
\int_{0}^{T} \int_{0}^{1} \int_{0}^{1} K_{N}(x, y) \partial_{x} \partial_{t} u^{N}(x, t) \partial_{x} \partial_{t} u^{N}(y, t) \mathrm{d} x \mathrm{~d} y \mathrm{~d} t=\int_{0}^{T} \sum_{n=0}^{N} r_{n} \sum_{i=0}^{2^{n}-1}\left(\int_{C_{i}^{n}} \partial_{x} \partial_{t} u^{N}(x, t) \mathrm{d} x\right)^{2} \mathrm{~d} t .
$$

Thus, using the $L^{2}$-orthogonal projection $P_{N}$ on $M_{N}$, the left-hand side of this inequality is equal to

$$
\int_{0}^{T} \sum_{n=0}^{N} r_{n} \sum_{i=0}^{2^{n}-1}\left(\int_{C_{i}^{n}} \partial_{x} \partial_{t} u^{N}(x, t) \mathrm{d} x\right)^{2}=\int_{0}^{T} \sum_{n=0}^{N} r_{n} h_{n}\left\|P_{n}\left(\partial_{x} \partial_{t} u^{N}(x, t)\right)\right\|_{L^{2}(0,1)}^{2} .
$$

Consequently if $\lim _{n \rightarrow+\infty} \frac{r_{n}}{2^{n}} \neq 0$, then it follows that $P_{N}\left(\partial_{x} \partial_{t} u^{N}(x, t)\right)$, which is equal to $\partial_{x} \partial_{t} u^{N}(x, t)$, is bounded in $L^{2}\left(0, T ; L^{2}(0,1)\right)$, independently of $N$, while for $\lim _{n \rightarrow+\infty} \frac{r_{n}}{2^{n}}=+\infty$, it means that $\partial_{x} \partial_{t} u^{N}(x, t) \rightarrow$ 0 strongly in $L^{2}\left(0, T ; L^{2}(0,1)\right)$ as $N$ tends to infinity.

Following this remark in the case $r_{n}=r_{0} \alpha^{n}$ with $\alpha \geq 2, \partial_{t x} u^{N}$ is bounded independently of $N$ in $L^{2}\left(0, T ; L^{2}(0,1)\right)$. Moreover if $\alpha>2, \partial_{t x} u^{N}$ tends strongly to zero in $L^{2}\left(0, T ; L^{2}(0,1)\right)$ and thus we obtain directly the

Proposition 2.8. In the case of a non convergent tree and under assumptions (24), (26), (27), and (25), $u^{N}$ tends to $u_{0}$ weakly in $L^{2}\left(0, T ; H_{0}^{1}(0,1)\right)$ as $N$ goes to infinity.

Proof. The proof is based on Proposition 1.9. In order to prove Proposition 2.8 we start by dividing the discrete weak formulation (35) by $\left\|K_{N}\right\|_{L^{1}(] 0,1[\times] 0,1[)}$. Since Lemma 1 is still valid, $u^{N}$ converges weakly $*$ in 
$L^{\infty}\left(0, T ; H_{0}^{1}(0,1)\right) \cap W^{1, \infty}\left(0, T ; L^{2}(0,1)\right)$ and strongly in $L^{2}\left(0, T ; L^{2}(0,1)\right)$. Consequently applying Proposition 1.9 , we can easily obtain that the limit $u$ of $u^{N}$ satisfies:

$$
\left\{\begin{array}{l}
-\int_{0}^{T} \int_{0}^{1} \partial_{x} u(x, t) \partial_{x t} \varphi(x, t) \mathrm{d} x \mathrm{~d} t=+\int_{0}^{1} \partial_{x} u_{0}(x) \partial_{x} \varphi(x, 0) \mathrm{d} x \mathrm{~d} y, \\
\forall \varphi \in \mathcal{D}\left(\left[0, T\left[; H_{0}^{1}(0,1)\right) .\right.\right.
\end{array}\right.
$$

Therefore $u=u_{0}$.

To obtain a non-trivial result in the limit, we should have assumed that the data satisfies other assumptions: for instance the load which applies on the mass-spring-tree chain goes to infinity like $\left\|K_{N}\right\|_{L^{1}(] 0,1[\times] 0,1[)}$ as $N$ goes to infinity.

\section{Conclusion, Future prospects}

We presented here a first step towards a general constitutive equation for the human lungs which takes into account the very nature of dissipation phenomena. In order to model realistic situations, this approach has to be extended to three-dimensional problems, and to integrate some new features. This extension raises some additional issues which are not only technical:

(1) The simple 1D spring/rigid mass system has to be replaced by a deformable medium, whose constitutive law may be complex due to the nature of the parenchyma, and may include extra dissipative effects.

(2) There is no canonical way to fill a given domain of $\mathbb{R}^{3}$ with the ends of an infinite dyadic tree. The way this filling is done in reality is only partially known, and it may vary from one individual to another. This problem has to be faced in order to build explicitly the mapping $\mathcal{R}$ in $3 \mathrm{D}$ domains.

Acknowledgements. We would like to acknowledge the support of ACI NIM project LePoumonVousDisJe, and the organizers of CEMRACS 2004, during which this work has been initiated.

\section{REFERENCES}

[1] H. Brezis, Analyse fonctionnelle. Théorie et applications. Masson (1993).

[2] C. Grandmont, Y. Maday and B. Maury, A multiscale/multimodel approach of the respiration tree, in Proc. of the International Conference, "New Trends in Continuum Mechanics" 8-12 September 2003, Constantza, Romania Theta Foundation Publications, Bucharest (2005).

[3] Q. Grimal, A. Watzky and S. Naili, A one-dimensional model for the propagation of pressure waves through the lung. J. Biomechanics 35 (2002) 1081-1089.

[4] J. Kaye, F.P. Primiano Jr. and D.N. Metaxas, A 3D virtual environment for modeling mechanical cardiopulmonary interactions. Med. Imag. An. 2 (1998) 169-195.

[5] Y. Lanir, Constitutive equations for the lung tissue. J. Biomech Eng. 105 (1983) 374-380.

[6] A. Lefebvre and B. Maury, Micro-macro modelling of arrays of spheres interacting through lubrication forces, Prépublication du Laboratoire de Mathématiques de l'Université Paris-Sud (2005) 46.

[7] J.L. Lions and E. Magenes, Non-homogeneous Boundary Value Problems and Applications, I. Springer-Verlag, New York (1972).

[8] G.N. Maksym and J.H.T. Bates, A distributed nonlinear model of lung tissue elasticity. J. Appl. Phys. 82 (1997) 32-41.

[9] B. Mauroy, M. Filoche, J.S. Andrade Jr. and B. Sapoval, Interplay between flow distribution and geometry in an airway tree. Phys. Rev. Lett. 14 (2003) 90.

[10] B. Mauroy, M. Filoche, E.R. Weibel and B. Sapoval, The optimal bronchial tree is dangerous. Nature 427 (2004) $633-636$.

[11] P. Oswald, Multilevel norms for $H^{-1 / 2}$. Computing 61 (1998) 235-255.

[12] S.B. Ricci, P. Cluzel, A. Constantinescu and T. Similowski, Mechanical model of the inspiratory pump. J. Biomechanics 35 (2002) 139-145.

[13] J.R. Rodarte, Stress-strain analysis and the lung. Fed. Proc. 41 (1982) 130-135. 